\title{
Renormalization of Hořava gravity
}

\author{
Andrei O. Barvinsky, ${ }^{1,2,3}$ Diego Blas, ${ }^{4}$ Mario Herrero-Valea, ${ }^{5,6}$ Sergey M. Sibiryakov, ${ }^{4,7,8}$ and Christian F. Steinwachs ${ }^{9}$ \\ ${ }^{1}$ Theory Department, Lebedev Physics Institute, Leninsky Prospect 53, Moscow 119991, Moscow, Russia \\ ${ }^{2}$ Department of Physics, Tomsk State University, Lenin Avenue 36, Tomsk 634050, Tomsk Oblast, Russia \\ ${ }^{3}$ Department of Physics and Astronomy, Pacific Institue for Theoretical Physics, \\ University of British Columbia, 6224 Agricultural Road, Vancouver, British Columbia V6T 1Z1, Canada \\ ${ }^{4}$ Theoretical Physics Department, CERN, CH-1211 Geneva 23, Geneva, Switzerland \\ ${ }^{5}$ Instituto de Física Teórica UAM/CSIC, C/ Nicolas Cabrera, 13-15, C.U. Cantoblanco, 28049 Madrid, \\ Community of Madrid, Spain \\ ${ }^{6}$ Departamento de Física Teórica, Universidad Autónoma de Madrid, \\ 20849 Madrid, Community of Madrid, Spain \\ ${ }^{7}$ Laboratory of Particle Physics and Cosmology, Institute of Physics, EPFL, CH-1015 Lausanne, \\ Vaud, Switzerland \\ ${ }^{8}$ Institute for Nuclear Research of the Russian Academy of Sciences, \\ 60th October Anniversary Prospect, 7a, 117312 Moscow, Moscow, Russia \\ ${ }^{9}$ Physikalisches Institut, Albert-Ludwigs-Universität Freiburg, Hermann-Herder-Strasse 3, \\ 79104 Freiburg, Baden-Württemberg, Germany
}

(Received 7 January 2016; published 8 March 2016)

\begin{abstract}
We prove perturbative renormalizability of projectable Hořava gravity. The key element of the argument is the choice of a gauge which ensures the correct anisotropic scaling of the propagators and their uniform falloff at large frequencies and momenta. This guarantees that the counterterms required to absorb the loop divergences are local and marginal or relevant with respect to the anisotropic scaling. Gauge invariance of the counterterms is achieved by making use of the background-covariant formalism. We also comment on the difficulties of this approach when addressing the renormalizability of the nonprojectable model.
\end{abstract}

DOI: 10.1103/PhysRevD.93.064022

\section{INTRODUCTION}

The construction of a consistent theory of quantum gravity has remained one of the major challenges in theoretical physics for many decades. String theory provides a fruitful approach to this problem, see e.g. [1], at the expense of introducing a very rich extra structure (and complexity) and it makes sense to question if other directions are possible. In particular, one may wonder whether gravity can be quantized in the framework of perturbative quantum field theory in four dimensions, as other fundamental forces in nature.

At low energies gravity is very well described by the Einstein-Hilbert action, which is perturbatively nonrenormalizable and therefore does not correspond to an ultraviolet (UV) complete theory (at least in perturbation theory). It has been known for several decades that a renormalizable theory is obtained by augmenting the action with quadratic curvature invariants [2]. For certain regions in the parameter space the theory is even asymptotically free and hence UV complete [3,4]. However, due to the presence of four time derivatives of the metric in the

Published by the American Physical Society under the terms of the Creative Commons Attribution 3.0 License. Further distribution of this work must maintain attribution to the author(s) and the published article's title, journal citation, and DOI.
Lagrangian, the theory contains ghosts-negative-norm states-and does not admit the usual interpretation along the lines of unitary quantum mechanics. ${ }^{1}$

An interesting development was proposed by $\mathrm{P}$. Hořava $[7,8]$, who pointed out that unitarity can be preserved at the expense of sacrificing the Lorentz invariance. In this case one can keep the action as second order in time derivatives, supplementing it only with terms containing higher spatial derivatives. This allows one to construct an action for gravity which is power-counting renormalizable, i.e. it contains only marginal and relevant operators with respect to the scaling transformations

$$
t \mapsto b^{-d} t, \quad x^{i} \mapsto b^{-1} x^{i}, \quad i=1, \ldots, d,
$$

where $b$ is an arbitrary scaling parameter and $d$ is the number of spatial dimensions. Note that time and space scale differently in (1). This type of transformation is called anisotropic scaling or Lifshitz scaling. The metric has zero scaling dimension under ${ }^{2}(1)$,

$$
\gamma_{i j} \mapsto \gamma_{i j}
$$

\footnotetext{
${ }^{1}$ See $[5,6]$ for a recent revival of this idea.

${ }^{2}$ To be precise, this applies to the spatial components of the metric; see Sec. II for details.
} 
and thus the nonlinearities of gravity do not give rise to any irrelevant interactions.

Hořava's proposal generated a surge of papers exploring its low-energy consistency and phenomenology; see $[9,10]$ for reviews. This led to the identification of a version of the proposal-the so-called healthy nonprojectable model [11] — which provides a consistent theory capable of reproducing the phenomenology of general relativity (GR) at the distance scales where the latter has been tested. It has also been realized that the theory never reduces to GR exactly: a certain amount of Lorentz invariance violation persists in the gravity sector at all energy/distance scales [12]. This can have interesting implications for cosmological models of dark energy [13]. Conservatively, one can use astrophysical and cosmological data to constrain the parameters of the theory [14-16]. Last but not least, to be phenomenologically viable, this scenario should be supplemented by a mechanism ensuring Lorentz invariance in the sector of visible matter where it has been tested with utmost precision. This represents a serious challenge that several proposals try to address [17-20].

Besides application to gravitation in four dimensions, it was suggested that Hořava gravity in $d=2$ can govern the dynamics of membranes in M-theory [7]. Other uses include the holographic description of nonrelativistic strongly coupled systems, analogous to those occurring in condensed matter physics [21,22].

Despite the vast literature on Hořava gravity, its renormalizability has not yet been rigorously proven. Indeed, while in pure scalar and fermionic Lifshitz theories with non-negative scaling dimensions of the fields ${ }^{3}$ renormalizability is a rather straightforward consequence of powercounting renormalizability [24]; this is not the case for gauge theories. As we explain below, a general local gauge fixing in Hořava gravity gives rise to certain "irregular" contributions in the propagator of the metric that may spoil the convergence of the loop integrals (see [25] for a similar phenomenon in nonrelativistic gauge theories). As a consequence, a loop diagram that by a scaling argument should be finite can actually diverge and generate a counterterm not expected from the naive power counting. Moreover, the irregular terms in the propagators can potentially lead to nonlocal divergences. Hence, the key question is whether there exists a class of gauges where all propagators are regular.

In this paper we answer this question in the affirmative for the case of projectable Hořava gravity. Unfortunately, this version of Hořava gravity does not reproduce GR at low energies (at least not within weak coupling) [12]. Nevertheless, it presents an interesting example of a theory sharing many properties of GR, such as a large gauge group of local spacetime transformations and the presence of

\footnotetext{
${ }^{3}$ In theories containing fields with negative dimensions the situation is more subtle [23].
}

gapless transverse-traceless (TT) excitations-gravitonsin dimensions $d=3$ and higher. Working in the gauge with regular propagators we demonstrate, with methods along the lines of relativistic gauge theories, that projectable Hořava gravity is perturbatively renormalizable in the strict sense.

In the nonprojectable case, we find that there is no gauge fixing which could remove all irregular contributions, though they can be reduced to only a few terms in the propagators for the lapse function [(00)-component of the metric]. Physically, these terms are a manifestation of the instantaneous interaction present in the theory $[12,26]$. We conclude that the renormalizability analysis in the nonprojectable case requires a careful treatment of the instantaneous mode.

Previous studies of the quantum properties of Horrava gravity span several directions. In Ref. [27] the projectable version in $d=3$ is considered with an additional restriction on the parameters imposed by the condition of detailed balance [8]. This model is connected to three-dimensional topologically massive gravity via the stochastic quantization approach and it is argued that it inherits the renormalizability properties of the latter. However, the treatment of the gauge invariance of Horava gravity in this construction is somewhat obscure. The works [28-31] explore the relation between Hořava gravity and causal dynamical triangulations. In Ref. [32] a one-loop renormalization was performed and the corresponding beta functions were computed in a truncated version of the $d=2$ projectable model. The truncation, however, explicitly breaks the gauge invariance of the theory. Finally, in Refs. [33] the one-loop counterterms for the gravitational effective action induced by a scalar field with Lifshitz scaling (see also [34-36] for earlier works on this subject) were computed. These counterterms were shown to have the same structure as the terms present in the bare action of Horava gravity, which suggests that if pure Hořava gravity is renormalizable, it remains so upon inclusion of matter. We return to this point in the conclusions (Sec. VII).

The paper is organized as follows. In Sec. II we introduce the projectable version of Hořava gravity in $(3+1)$ and $(2+1)$ dimensions. The $d=2$ case provides the simplest example of gravity with anisotropic scaling, which we use to illustrate the main ideas of our approach. In Sec. III we discuss the irregular terms arising in the propagators for a generic choice of gauge and the associated problems in the renormalization analysis. In Sec. IV we present a twoparameter family of gauges where the propagators are free from irregular contributions. Using this class of regular gauges we evaluate the degree of divergence of a generic diagram in Sec. V and argue that only local counterterms that are relevant or marginal with respect to the anisotropic scaling are required to renormalize the theory. By embedding our gauge-fixing procedure into the background-field formalism, we ensure that the counterterms preserve gauge 
invariance, which completes the proof of renormalizability. In Sec. VI we analyze the nonprojectable case and identify irregular contributions that cannot be removed by a gauge fixing. We conclude in Sec. VII. Some details of the derivations are relegated to the appendixes.

\section{PROJECTABLE HOŘAVA GRAVITY}

Geometrically, Hořava gravity differs from GR by the introduction of a preferred spacetime foliation by spacelike surfaces. The spacetime metric is represented using the Arnowitt-Deser-Misner (ADM) decomposition,

$$
\begin{aligned}
\mathrm{d} s^{2} & =N^{2} \mathrm{~d} t^{2}-\gamma_{i j}\left(\mathrm{~d} x^{i}+N^{i} \mathrm{~d} t\right)\left(\mathrm{d} x^{j}+N^{j} \mathrm{~d} t\right), \\
i, j & =1, \ldots, d .
\end{aligned}
$$

We aim to construct the theory which is invariant under the subgroup of diffeomorphisms that preserve the foliation structure (FDiffs). These consist of time-dependent transformations of the spatial coordinates and space-independent reparametrizations of time,

$$
x^{i} \mapsto \tilde{x}^{i}(\mathbf{x}, t), \quad t \mapsto \tilde{t}(t),
$$

where $\tilde{t}(t)$ is a monotonic function. Under this symmetry the lapse $N$, the shift $N^{i}$ and the spatial metric $\gamma_{i j}$ transform in the standard way,

$$
\begin{aligned}
N & \mapsto \tilde{N}=N \frac{\mathrm{d} t}{\mathrm{~d} \tilde{t}}, \\
N^{i} & \mapsto \tilde{N}^{i}=\left(N^{j} \frac{\partial \tilde{x}^{i}}{\partial x^{j}}-\frac{\partial \tilde{x}^{i}}{\partial t}\right) \frac{\mathrm{d} t}{\mathrm{~d} \tilde{t}}, \\
\gamma_{i j} & \mapsto \tilde{\gamma}_{i j}=\gamma_{k l} \frac{\partial x^{k}}{\partial \tilde{x}^{i}} \frac{\partial x^{l}}{\partial \tilde{x}^{j}} .
\end{aligned}
$$

We also impose time-reversal invariance, under which $N$ and $\gamma_{i j}$ are even, whereas the shift $N^{i}$ is odd.

We assign the following scaling dimensions to the fields according to their transformation under the anisotropic scaling $^{4}(1)$

$$
[N]=\left[\gamma_{i j}\right]=0, \quad\left[N^{i}\right]=d-1 .
$$

The action is constructed from local operators that transform as scalars under FDiffs and have dimension up to $2 d$,

$$
S=\frac{1}{2 \varkappa^{2}} \int \mathrm{d} t \mathrm{~d}^{d} x \sqrt{\gamma} N\left(K_{i j} K^{i j}-\lambda K^{2}-\mathcal{V}\right) .
$$

Here, $\varkappa^{2}, \lambda$ are free parameters and the extrinsic curvature of the foliation leaves is given by

\footnotetext{
${ }^{4}$ We assign dimension -1 to the spatial coordinates $x^{i}$. Accordingly, time has dimension $-d$. A field $\Phi$ with dimension $r$ transforms under the scaling (1) as $\Phi \mapsto b^{r} \Phi$.
}

$$
K_{i j}=\frac{\dot{\gamma}_{i j}-\nabla_{i} N_{j}-\nabla_{j} N_{i}}{2 N} .
$$

The trace is defined as $K=\gamma^{i j} K_{i j}$. The dot stands for a time derivative, indices are raised and lowered by the spatial metric $\gamma_{i j}$ and the covariant spatial derivatives $\nabla_{i}$ are compatible with $\gamma_{i j}$. The potential term $\mathcal{V}$ consists of all allowed combinations of local invariants of scaling dimension up to $2 d$ that are made of $\gamma_{i j}, N$ and their derivatives with respect to $\nabla_{i}$. In this way one obtains a Lagrangian consisting of marginal and relevant operators with respect to the anisotropic scaling which in this sense is powercounting renormalizable.

In the nonprojectable Hořava gravity the lapse $N$ is assumed to be a function of both space and time; we postpone the discussion of this case until Sec. VI. For the time being we focus on the projectable model where the lapse is a function of time only, $N=N(t)$. Then the time reparametrizations allow one to set $N=1$ leaving the timedependent spatial diffeomorphisms as the remaining gauge transformations.

In $d=3$, upon using the Bianchi identities and integration by parts, one finds the most general potential [37],

$$
\begin{aligned}
\mathcal{V}^{d=3}= & 2 \Lambda-\eta R+\mu_{1} R^{2}+\mu_{2} R_{i j} R^{i j}+\nu_{1} R^{3}+\nu_{2} R R_{i j} R^{i j} \\
& +\nu_{3} R_{j}^{i} R_{k}^{j} R_{i}^{k}+\nu_{4} \nabla_{i} R \nabla^{i} R+\nu_{5} \nabla_{i} R_{j k} \nabla^{i} R^{j k}
\end{aligned}
$$

Here, $R_{i j}$ and $R$ are the Ricci tensor and Ricci scalar constructed from $\gamma_{i j}$. In total, the theory contains 11 couplings: $\varkappa^{2}, \lambda, \Lambda, \eta, \mu_{1,2}$ and $\nu_{a}, a=1, \ldots, 5$. The terms with coefficients $\nu_{a}, a=1, \ldots, 5$ in (4) together with the extrinsic-curvature terms in (3) are marginal under the scaling (1). They determine the UV behavior of the theory, in particular its renormalizability properties. The rest of the terms in (4) are relevant deformations. Among them is the cosmological constant $\Lambda$, which has the lowest dimension. We assume that it is tuned to zero in order to admit flat Minkowski spacetime as a solution.

Let us study the spectrum of linear perturbations around this background. We write

$$
\gamma_{i j}=\delta_{i j}+h_{i j}
$$

and decompose the perturbations into scalar, vector and TT tensor parts,

$N^{i}=\partial_{i} B+u_{i}$,

$h_{i j}=\left(\delta_{i j}-\frac{\partial_{i} \partial_{j}}{\Delta}\right) \psi+\frac{\partial_{i} \partial_{j}}{\Delta} E+\partial_{i} v_{j}+\partial_{j} v_{i}+\zeta_{i j}$

with

$$
\partial_{i} u_{i}=\partial_{i} v_{i}=\partial_{i} \zeta_{i j}=\zeta_{i i}=0 .
$$


Here $\Delta$ is the flat-space Laplacian. The quadratic action reads

$$
\begin{aligned}
S_{2}^{d=3}= & \frac{1}{2 \varkappa^{2}} \int \mathrm{d} t \mathrm{~d}^{3} x\left[\frac{\dot{\zeta}_{i j}^{2}}{4}+\frac{\eta}{4} \zeta_{i j} \Delta \zeta_{i j}-\frac{\mu_{2}}{4} \zeta_{i j} \Delta^{2} \zeta_{i j}\right. \\
& +\frac{\nu_{5}}{4} \zeta_{i j} \Delta^{3} \zeta_{i j}-\frac{1}{2}\left(\dot{v}_{i}-u_{i}\right) \Delta\left(\dot{v}_{i}-u_{i}\right) \\
& +\frac{\dot{\psi}^{2}}{2}+\frac{1}{4}(\dot{E}-2 \Delta B)^{2}-\frac{\lambda}{4}(2 \dot{\psi}+\dot{E}-2 \Delta B)^{2} \\
& -\frac{\eta}{2} \psi \Delta \psi-\left(4 \mu_{1}+\frac{3 \mu_{2}}{2}\right) \psi \Delta^{2} \psi \\
& \left.+\left(4 \nu_{4}+\frac{3 \nu_{5}}{2}\right) \psi \Delta^{3} \psi\right] .
\end{aligned}
$$

In order to identify the physical degrees of freedom we perform the variation with respect to $u_{i}$ and $B$ and set them to zero afterwards by the gauge choice. We obtain the equations

$$
\Delta \dot{v}_{i}=0, \quad \Delta\left(\dot{E}-\frac{2 \lambda}{1-\lambda} \dot{\psi}\right)=0
$$

The first one implies that the vector sector does not contain any propagating modes. From the second equation in (7) we express $\dot{E}$ and substitute it back into (6) which yields the action for the propagating degrees of freedom,

$$
\begin{aligned}
S_{2}^{d=3}= & \frac{1}{2 \varkappa^{2}} \int \mathrm{d} t \mathrm{~d}^{3} x\left[\frac{\dot{\zeta}_{i j}^{2}}{4}+\frac{\eta}{4} \zeta_{i j} \Delta \zeta_{i j}-\frac{\mu_{2}}{4} \zeta_{i j} \Delta^{2} \zeta_{i j}\right. \\
& +\frac{\nu_{5}}{4} \zeta_{i j} \Delta^{3} \zeta_{i j}+\frac{1-3 \lambda}{2(1-\lambda)} \dot{\psi}^{2}-\frac{\eta}{2} \psi \Delta \psi \\
& \left.-\left(4 \mu_{1}+\frac{3 \mu_{2}}{2}\right) \psi \Delta^{2} \psi+\left(4 \nu_{4}+\frac{3 \nu_{5}}{2}\right) \psi \Delta^{3} \psi\right] .
\end{aligned}
$$

In addition to the TT mode $\zeta_{i j}$, the theory propagates a "scalar graviton" $\psi$. Both modes have positive-definite kinetic terms provided $\varkappa^{2}>0$ and $\lambda$ is either smaller than $1 / 3$ or larger than 1 . The dispersion relations of the two modes are respectively

$\omega_{t t}^{2}=\eta k^{2}+\mu_{2} k^{4}+\nu_{5} k^{6}$,

$\omega_{s}^{2}=\frac{1-\lambda}{1-3 \lambda}\left(-\eta k^{2}+\left(8 \mu_{1}+3 \mu_{2}\right) k^{4}+\left(8 \nu_{4}+3 \nu_{5}\right) k^{6}\right)$.

This immediately raises a problem: the term proportional to $k^{2}$ in the dispersion relation cannot be positive for both modes simultaneously. Thus, nonzero $\eta$ leads to an instability of the Minkowski background with respect to inhomogeneous perturbations. For positive values of the parameters $\mu_{1,2}$ and $\nu_{4,5}$ the instability is cut off at large spatial momenta and therefore does not affect the UV properties of the theory. Moreover, we can stabilize the Minkowski spacetime by simply tuning $\eta$ to zero. However, in that case the dispersion relations of the TT mode and scalar gravitons are quadratic, $\omega \propto k^{2}$, down to zero momentum, which prevents recovery of GR at low energies. ${ }^{5}$

The situation is much simpler for $d=2$. In this case the potential includes only two terms,

$$
\mathcal{V}^{d=2}=2 \Lambda+\mu R^{2} .
$$

The linear in $R$ term is absent because the combination $\sqrt{\gamma} R$ is a total derivative in two dimensions. Also the Ricci tensor $R_{i j}$ reduces to the scalar curvature, so the invariant $R_{i j} R^{i j}$ is proportional to $R^{2}$. Setting the cosmological constant $\Lambda$ to zero, we obtain a model with three marginal couplings: $\varkappa^{2}, \lambda$ and $\mu$.

The spectrum of this model is derived along the same lines as for the $d=3$ case. Expanding around flat spacetime and performing the decomposition (5) - where now the TT component $\zeta_{i j}$ is absent-we obtain the quadratic action,

$$
\begin{aligned}
S_{2}^{d=2}= & \frac{1}{2 \varkappa^{2}} \int \mathrm{d} t \mathrm{~d}^{2} x\left[-\frac{1}{2}\left(\dot{v}_{i}-u_{i}\right) \Delta\left(\dot{v}_{i}-u_{i}\right)+\frac{\dot{\psi}^{2}}{4}\right. \\
& \left.+\frac{1}{4}(\dot{E}-2 \Delta B)^{2}-\frac{\lambda}{4}(\dot{\psi}+\dot{E}-2 \Delta B)^{2}-\mu \psi \Delta^{2} \psi\right] .
\end{aligned}
$$

We observe that the action for the vector perturbations has exactly the same structure as in $d=3$, implying that there are no propagating modes in this sector. In the scalar sector we eliminate $E$ using the equation obtained upon variation with respect to $B$ and set $B=0$ afterwards. This yields

$$
S_{2}^{d=2}=\frac{1}{2 \varkappa^{2}} \int \mathrm{d} t \mathrm{~d}^{2} x\left[\frac{1-2 \lambda}{4(1-\lambda)} \dot{\psi}^{2}-\mu \psi \Delta^{2} \psi\right] .
$$

Unlike GR, which in $(2+1)$ dimensions does not possess any local degrees of freedom, Hořava gravity propagates a dynamical scalar mode. The latter has the dispersion relation

$$
\omega_{s}^{2}=4 \mu \frac{1-\lambda}{1-2 \lambda} k^{4} .
$$

It is well behaved (i.e. has positive kinetic term and is stable) if $\varkappa^{6}>0, \mu>0$ and $\lambda<1 / 2$ or $\lambda>1$. We make extensive use of the $d=2$ model in what follows.

\footnotetext{
${ }^{5}$ One could try to keep $\eta$ finite and positive and suppress the instability associated to the scalar graviton by tuning $\lambda$ close to 1 . However, in this limit the theory becomes strongly coupled and the perturbative treatment breaks down $[12,38]$.

${ }^{6}$ We take $\varkappa^{2}>0$ to make contact with higher dimensions where this condition is required for positivity of the TT mode kinetic energy.
} 
In order to analyze the renormalizability properties of the theory, from now on we transform to "Euclidean" time by the Wick rotation

$$
t \mapsto \tau=i t, \quad N^{j} \mapsto N_{E}^{j}=-i N^{j}
$$

In the following we omit the subscript " $E$ " on the Euclidean shift. The corresponding action differs from (3) only by the sign of the potential term. At the quadratic level this amounts to flipping the signs of the terms containing $\mu_{1,2}, \nu_{4,5}$ in (6) and of the $\mu$-term in (11).

\section{LOCAL GAUGE FIXING AND IRREGULAR TERMS}

In this section we focus on the theory in $d=2$. In order to quantize the theory we need to fix the gauge. Finding a suitable gauge turns out to be nontrivial, as we demonstrate below. The technical part of the following analysis is straightforward. Upon adding a gauge-fixing term to the quadratic action (11) and transforming to momentum space, we invert the kinetic matrices for the scalar and vector perturbations. The propagators of the shift and the spatial metric are then reconstructed from these helicity components using Eqs. (5),

$$
\begin{aligned}
\left\langle N^{i}(p) N^{j}(-p)\right\rangle= & \left\langle u_{i} u_{j}\right\rangle+k_{i} k_{j}\langle B B\rangle, \\
\left\langle N^{i}(p) h_{j k}(-p)\right\rangle= & -i k_{j}\left\langle u_{i} v_{k}\right\rangle-i k_{k}\left\langle u_{i} v_{j}\right\rangle \\
& +i k_{i}\left(\delta_{j k}-\hat{k}_{j} \hat{k}_{k}\right)\langle B \psi\rangle+i k_{i} \hat{k}_{j} \hat{k}_{k}\langle B E\rangle .
\end{aligned}
$$

$$
\begin{aligned}
\left\langle h_{i j}(p) h_{k l}(-p)\right\rangle= & k_{i} k_{k}\left\langle v_{j} v_{l}\right\rangle+k_{j} k_{k}\left\langle v_{i} v_{l}\right\rangle+k_{i} k_{l}\left\langle v_{j} v_{k}\right\rangle \\
& +k_{j} k_{l}\left\langle v_{i} v_{k}\right\rangle+\left(\delta_{i j}-\hat{k}_{i} \hat{k}_{j}\right)\left(\delta_{k l}-\hat{k}_{k} \hat{k}_{l}\right)\langle\psi \psi \psi\rangle \\
& +\left(\delta_{i j}-\hat{k}_{i} \hat{k}_{j}\right) \hat{k}_{k} \hat{k}_{l}\langle\psi E\rangle \\
& +\hat{k}_{i} \hat{k}_{j}\left(\delta_{k l}-\hat{k}_{k} \hat{k}_{l}\right)\langle E \psi\rangle+\hat{k}_{i} \hat{k}_{j} \hat{k}_{k} \hat{k}_{l}\langle E E\rangle .
\end{aligned}
$$

Here we introduced the notations

$$
p \equiv(\omega, \mathbf{k}), \quad \hat{k}_{i} \equiv k_{i} / k
$$

We postpone the discussion of the Faddeev-Popov ghosts coming from the gauge fixing to Sec. IVA 1.

Let us illustrate the type of problems connected to the gauge-fixing procedure by considering as a first trial the gauge

$$
N^{i}=0 .
$$

It can be implemented by adding the term

$$
\mathcal{L}_{g f}=\frac{\sigma}{2 \varkappa^{2}}\left(N^{i}\right)^{2}
$$

to the Lagrangian and taking the limit $\sigma \rightarrow \infty$. Alternatively, one can simply set $u_{i}=B=0$ in (11). The kinetic matrix for the remaining variables is now invertible yielding the propagators

$$
\begin{aligned}
& \left\langle v_{i}(p) v_{j}(-p)\right\rangle=\frac{2 \varkappa^{2}}{\omega^{2} k^{2}}\left(\delta_{i j}-\hat{k}_{i} \hat{k}_{j}\right), \\
& \langle\psi(p) \psi(-p)\rangle=\frac{4 \varkappa^{2}(1-\lambda)}{1-2 \lambda} \mathcal{P}_{s}(p), \\
& \langle\psi(p) E(-p)\rangle=\frac{4 \varkappa^{2} \lambda}{1-2 \lambda} \mathcal{P}_{s}(p), \\
& \langle E(p) E(-p)\rangle=\frac{4 \varkappa^{2} \lambda^{2}}{(1-\lambda)(1-2 \lambda)} \mathcal{P}_{s}(p)+\frac{4 \varkappa^{2}}{(1-\lambda) \omega^{2}},
\end{aligned}
$$

where

$$
\mathcal{P}_{s}(p)=\left[\omega^{2}+4 \mu \frac{1-\lambda}{1-2 \lambda} k^{4}\right]^{-1}
$$

has the pole corresponding to the physical mode. ${ }^{7}$ Note the presence of the transverse projector in (14a) which is implied by the transversality of $v_{i}$. Substituting these expressions into (12c) we obtain

$$
\begin{aligned}
\left\langle h_{i j}(p) h_{k l}(-p)\right\rangle= & \frac{4 \varkappa^{2}(1-\lambda)}{1-2 \lambda} \delta_{i j} \delta_{k l} \mathcal{P}_{s}(p) \\
& +\left(\delta_{i k} \delta_{j l}+\delta_{i l} \delta_{j k}-2 \delta_{i j} \delta_{k l}\right) \frac{2 \varkappa^{2}}{\omega^{2}} \\
& +16 \varkappa^{2} \mu\left[\frac{1-\lambda}{1-2 \lambda}\left(\delta_{i j} k_{k} k_{l}+k_{i} k_{j} \delta_{k l}\right) k^{2}\right. \\
& \left.-k_{i} k_{j} k_{k} k_{l}\right] \frac{\mathcal{P}_{s}(p)}{\omega^{2}}
\end{aligned}
$$

In deriving this expression we used the dimensional dependent identity (A1) that can be found in Appendix A.

We observe that besides the first contribution proportional to $\mathcal{P}_{s}(p)$, which uniformly decreases whenever $\omega$ or $k$ goes to infinity, the propagator (16) contains terms of the form $1 / \omega^{2}$ and $O\left(k^{4}\right) \mathcal{P}_{s} / \omega^{2}$ that do not fall off with the spatial momentum. The latter terms are dangerous as they lead to nonlocal singularities of the propagator in position space. For example, the Fourier transform of the second term in (16) has the form

\footnotetext{
${ }^{7}$ Recall that we are working in the Euclidean time, so the sign of the $\mu$-term in (11) must be flipped to " + ."
} 


$$
\left\langle h_{i j}(\tau, \mathbf{x}) h_{k l}(0)\right\rangle \ni-\varkappa^{2}\left(\delta_{i k} \delta_{j l}+\delta_{i l} \delta_{j k}-2 \delta_{i j} \delta_{k l}\right)|\tau| \delta^{(2)}(\mathbf{x}),
$$

where $\delta^{(2)}$ is the $\delta$-function. This is singular at $\mathbf{x}=0$ for all times $\tau .{ }^{8}$ In the perturbative expansion such contributions will give rise to overlapping singularities that are nonlocal in time. In the more familiar momentum-space representation they correspond to divergences in the loop diagrams which have a nonpolynomial dependence on the external frequency. Unless these divergences cancel order by order of perturbation theory, they jeopardize the renormalizability by requiring the introduction of counterterms with nonlocal time dependence. ${ }^{9}$ Clearly, even if the cancellation of nonlocal divergences does take place, it will be increasingly hard to keep track of it at higher loop orders. Thus, we conclude that the gauge (13) is not suitable for the analysis of renormalizability.

The gauge (13) is rather special and one might think that the nonlocal singularities in the propagators can be avoided once we allow for a more general gauge-fixing condition. Let us now show that this is not the case as long as one restricts to local gauge-fixing terms. The most general term of this class has the form

$$
\mathcal{L}_{g f}=\frac{\sigma}{2 \varkappa^{2}} F^{i} \mathcal{O}_{i j} F^{j}
$$

where $F^{i}$ is a linear combination of the fields $N^{i}, h_{i j}$ and their derivatives which transforms as a vector under spatial rotations, while $\mathcal{O}_{i j}$ is an invertible local operator. In order not to spoil the scaling properties of the action, the gaugefixing term should not introduce any dimensionful couplings with respect to the scaling (1). This implies that the total dimension of $\mathcal{L}_{g f}$ must be 4 , whereas all terms in $F^{i}$ and $\mathcal{O}_{i j}$ must scale in the same way. A local operator $\mathcal{O}_{i j}$ can contain only the identity and a finite number of derivatives, and therefore its scaling dimension is non-negative. This implies that the dimension of $F^{i}$ must

\footnotetext{
${ }^{8}$ On the contrary, the Fourier transform of $\mathcal{P}_{s}$ is singular only at $\tau=\mathbf{x}=0$. This is explicitly verified using the representation

$$
\begin{aligned}
& \int \frac{\mathrm{d} \omega \mathrm{d}^{2} k}{(2 \pi)^{3}} \frac{\mathrm{e}^{-i \omega \tau+i k x}}{\omega^{2}+A^{2} k^{4}}=-\frac{1}{\Delta} \int \frac{\mathrm{d}^{2} k}{(2 \pi)^{2} A} \mathrm{e}^{-A k^{2}|\tau|+i k x} \\
& =\frac{-1}{16 \pi^{2} A^{2}|\tau|} \int \mathrm{d}^{2} y \log |\mathbf{x}-\mathbf{y}| \exp \left[-\frac{y^{2}}{4 A|\tau|}\right] .
\end{aligned}
$$

The rhs is smooth together with all its derivatives whenever $\tau$ or $\mathbf{x}$ is nonzero.

${ }^{9} \mathrm{~A}$ hint towards such cancellation comes from considering one-graviton exchange between two external sources. One can check that the irregular contributions drop off from this amplitude if the sources are conserved, as required by FDiff invariance. This argument is not directly applicable at higher orders of perturbation theory where the sources coupled to the metric are not conserved due to the nonlinearities of the theory.
}

be less than or equal to 2 . This excludes that $F^{i}$ can contain time derivatives of the shift, since such terms would already have at least a scaling dimension of 3 . The time derivative of $h_{i j}$ also cannot appear in $F^{i}$ because to obtain from it an object with a single index one must introduce an additional spatial derivative, which again raises the dimension up to 3. Finally, $F^{i}$ cannot contain $N^{i}$ and the spatial derivatives of the metric $h_{i j}$ simultaneously, as otherwise it would explicitly break the time-reversal invariance. ${ }^{10}$ Thus we arrive at two possibilities:

$$
F^{i}=N^{i} \quad \text { or } \quad F^{i}=\partial_{j} h_{i j}+\sigma^{\prime} \partial_{i} h,
$$

where $\sigma^{\prime}$ is an arbitrary coefficient and $h$ is the trace of $h_{i j}$. Both these combinations have dimension 1, so the corresponding operator $\mathcal{O}_{i j}$ must be of dimension 2. Hence, it has the form

$$
\mathcal{O}_{i j}=-\delta_{i j} \Delta-\sigma^{\prime \prime} \partial_{i} \partial_{j}
$$

Without delving into the study of the full propagators, let us focus on the transverse component $u_{i}$ of the shift. For the first choice of the gauge-fixing function in (18) a straightforward calculation yields

$$
\left\langle u_{i}(p) u_{j}(-p)\right\rangle=\frac{\varkappa^{2}}{\sigma k^{2}}\left(\delta_{i j}-\hat{k}_{i} \hat{k}_{j}\right)
$$

whereas for the second choice one obtains

$$
\left\langle u_{i}(p) u_{j}(-p)\right\rangle=\left[\frac{2 \varkappa^{2}}{k^{2}}+\frac{\varkappa^{2} \omega^{2}}{\sigma k^{6}}\right]\left(\delta_{i j}-\hat{k}_{i} \hat{k}_{j}\right) .
$$

In both cases the propagator contains contributions independent of the frequency that behave as $1 / k^{2}$. This, in turn, leads to a nonlocal singularity of the $\left\langle N^{i} N^{j}\right\rangle$ propagator in the position space proportional to

$$
\delta^{(1)}(\tau) \frac{1}{4 \pi} \log |\mathbf{x}| .
$$

In perturbation theory this will produce spurious divergences that are nonlocal in space. Therefore, none of the local gauges (18) is appropriate for our purposes.

\section{REGULAR GAUGES}

Let us introduce some terminology: consider two fields $\Phi_{1}, \Phi_{2}$ that have scaling dimensions $r_{1}, r_{2}$ under (1). Following [25] we denote the propagator $\left\langle\Phi_{1} \Phi_{2}\right\rangle$ regular if it is given by the sum of terms of the form,

\footnotetext{
${ }^{10}$ Note that in $d=3$ such a combination is further forbidden by the mismatch between the scaling dimensions of the shift $N^{i}$ and of the spatial derivatives of $h_{i j}$.
} 


$$
\frac{P(\omega, \mathbf{k})}{D(\omega, \mathbf{k})}
$$

where $D$ is a product of monomials,

$D=\prod_{m=1}^{M}\left(A_{m} \omega^{2}+B_{m} k^{2 d}+\cdots\right), \quad A_{m}, B_{m}>0$,

and $P(\omega, \mathbf{k})$ is a polynomial of scaling degree ${ }^{11}$ less than or equal to $r_{1}+r_{2}+2(M-1) d$. We emphasize that all constants $A_{m}, B_{m}$ in (19b) must be strictly positive. The ellipsis stands for terms with lower scaling dimensions that generically arise in theories with relevant operators in the action. The reader will easily convince herself that a regular propagator has only local singularities at $\tau=\mathbf{x}=0$ in position space. Due to the restriction on the degree of the numerator it scales at short distances and time intervals as

$$
\left\langle\Phi_{1}\left(b^{-d} \tau, b^{-1} \mathbf{x}\right) \Phi_{2}(0)\right\rangle=b^{r_{1}+r_{2}}\left\langle\Phi_{1}(\tau, \mathbf{x}) \Phi_{2}(0)\right\rangle
$$

The results of the previous section show that in order to obtain regular propagators in Hořava gravity we need to go beyond local gauge-fixing terms.

\section{A. Theory in two spatial dimensions}

As a starting point and for guidance in the treatment of nonrelativistic theories, let us first review the structure of covariant gauges in relativistic theories. In GR and its higher-derivative extensions the corresponding gaugefixing Lagrangians can be adjusted such as to cancel the terms mixing different metric components in the quadratic action. Such a gauge fixing renders the tensor structure of the propagators diagonal and greatly simplifies the actual computations. The spatial diffeomorphisms are fixed in the covariant gauges by the conditions $F^{i}=0$. In terms of the ADM variables they have the general form

$$
F^{i}=\dot{N}^{i}-\partial_{j} h_{i j}-C \partial_{i}(2 \phi+h),
$$

where $\phi$ is the perturbation of the lapse and $C$ is a numerical constant. Such a form is not appropriate for Hořava gravity, because $\dot{N}^{i}$ and spatial derivatives of $h_{i j}$ have different scaling dimensions. The discrepancy is easily compensated by introducing two more spatial derivatives acting on the spatial metric. Thus, for $d=2$ Hořava gravity we consider the gauge-fixing function of the general form,

$$
F^{i}=\dot{N}^{i}-C_{1} \Delta \partial_{j} h_{i j}-C_{2} \Delta \partial_{i} h-C_{3} \partial_{i} \partial_{j} \partial_{k} h_{j k}
$$

The coefficients $C_{1,2,3}$ are chosen shortly to simplify the quadratic action. Note the presence of the last term in (21) which does not have an analog in the relativistic case (20).

\footnotetext{
${ }^{11}$ The scaling degree of a polynomial is defined as the maximal scaling dimension of its terms.
}

Importantly, with the gauge-fixing function (21) the operator $\mathcal{O}_{i j}$ in the gauge-fixing Lagrangian (17) must have dimension -2 and thus is necessarily nonlocal. We take

$\mathcal{O}_{i j}=-\left[\delta_{i j} \Delta+\xi \partial_{i} \partial_{j}\right]^{-1}=-\frac{\delta_{i j}}{\Delta}+\frac{\xi}{(1+\xi)} \frac{\partial_{i} \partial_{j}}{\Delta^{2}}$

Though unusual, the nonlocality of the gauge-fixing Lagrangian does not introduce any problems in the perturbative expansion around flat spacetime, as it appears only in the quadratic action. ${ }^{12}$ The only important property at this point is the invertibility of $\mathcal{O}_{i j}$.

We now choose the coefficients in the linear combination (21) in such a way that the contributions coming from $\mathcal{L}_{g f}$ cancel the terms mixing $N^{i}$ and $h_{i j}$ in the quadratic Lagrangian. The combination with the required properties is

$$
\begin{aligned}
F^{i} & =\dot{N}^{i}+\frac{1}{2 \sigma} \mathcal{O}_{i j}^{-1} \partial_{k} h_{j k}-\frac{\lambda}{2 \sigma} \mathcal{O}_{i j}^{-1} \partial_{j} h \\
& =\dot{N}^{i}-\frac{1}{2 \sigma} \Delta \partial_{k} h_{i k}+\frac{\lambda(1+\xi)}{2 \sigma} \Delta \partial_{i} h-\frac{\xi}{2 \sigma} \partial_{i} \partial_{j} \partial_{k} h_{j k} .
\end{aligned}
$$

In this way we arrive at a two-parameter family of gauges depending on $\sigma$ and $\xi$. It is instructive to write down the total quadratic Lagrangian in this $\sigma \xi$-gauge,

$$
\begin{aligned}
\mathcal{L}_{2}^{d=}=2 & +\mathcal{L}_{g f} \\
= & \frac{1}{2 \varkappa^{2}}\left[\frac{\dot{h}_{i j}^{2}}{4}-\frac{\lambda \dot{h}^{2}}{4}-\frac{1}{4 \sigma} \partial_{j} h_{i j} \Delta \partial_{k} h_{i k}+\left(\mu+\frac{\xi}{4 \sigma}\right)\left(\partial_{i} \partial_{j} h_{i j}\right)^{2}\right. \\
& -\left(2 \mu+\frac{\lambda(1+\xi)}{2 \sigma}\right) \Delta h \partial_{i} \partial_{j} h_{i j}+\left(\mu+\frac{\lambda^{2}(1+\xi)}{4 \sigma}\right)(\Delta h)^{2} \\
& \left.-\sigma \dot{N}^{i}\left[\delta_{i j} \Delta+\xi \partial_{i} \partial_{j}\right]^{-1} \dot{N}^{j}+\frac{\left(\partial_{i} N^{j}\right)^{2}}{2}+\left(\frac{1}{2}-\lambda\right)\left(\partial_{i} N^{i}\right)^{2}\right] .
\end{aligned}
$$

Note that the nonlocality persists only in the term involving time derivatives of the shift.

Inserting again the helicity decomposition (5) into the above Lagrangian, inverting the operators that appear in the resulting quadratic forms, and combining all contributions in (12) we obtain the propagators

$$
\begin{aligned}
\left\langle N^{i}(p) N^{j}(-p)\right\rangle= & \frac{\varkappa^{2}}{\sigma}\left(k^{2} \delta_{i j}-k_{i} k_{j}\right) \mathcal{P}_{1}(p) \\
& +\frac{\varkappa^{2}(1+\xi)}{\sigma} k_{i} k_{j} \mathcal{P}_{2}(p),
\end{aligned}
$$

\footnotetext{
${ }^{12}$ It will require a careful treatment, however, when we generalize our analysis to the background-field formalism in Sec. V B.
} 


$$
\begin{aligned}
\left\langle h_{i j}(p) h_{k l}(-p)\right\rangle= & 4 \varkappa^{2} \delta_{i j} \delta_{k l}\left[\frac{1-\lambda}{1-2 \lambda} \mathcal{P}_{s}(p)-\mathcal{P}_{1}(p)\right] \\
& +2 \varkappa^{2}\left(\delta_{i k} \delta_{j l}+\delta_{i l} \delta_{j k}\right) \mathcal{P}_{1}(p) \\
& +4 \varkappa^{2}\left(\delta_{i j} \hat{k}_{k} \hat{k}_{l}+\hat{k}_{i} \hat{k}_{j} \delta_{k l}\right)\left[\mathcal{P}_{1}(p)-\mathcal{P}_{s}(p)\right] \\
& +4 \varkappa^{2} \hat{k}_{i} \hat{k}_{j} \hat{k}_{k} \hat{k}_{l}\left[\frac{1-2 \lambda}{1-\lambda} \mathcal{P}_{s}(p)\right. \\
& \left.-2 \mathcal{P}_{1}(p)+\frac{\mathcal{P}_{2}(p)}{1-\lambda}\right],
\end{aligned}
$$

whereas $\left\langle N^{i} h_{j k}\right\rangle$ trivially vanishes. Here $\mathcal{P}_{s}$ is given by the expression (15) and

$$
\begin{aligned}
& \mathcal{P}_{1}(p)=\left[\omega^{2}+\frac{k^{4}}{2 \sigma}\right]^{-1}, \\
& \mathcal{P}_{2}(p)=\left[\omega^{2}+\frac{(1-\lambda)(1+\xi)}{\sigma} k^{4}\right]^{-1} .
\end{aligned}
$$

In deriving Eq. (25b) we again made use of the identity (A1). The above propagators are regular in the sense of (19a) provided $^{13}$

$$
\sigma>0, \quad(1-\lambda)(1+\xi)>0 .
$$

Indeed, (25a) and the first two terms in (25b) are obviously regular. For the terms in the second line of (25b) the situation is subtler. One may worry that the longitudinal projectors entering them contain factors $k^{2}$ in the denominator and apparently violate the regular form (19b). However, we observe that the combinations in the square brackets in these terms vanish at $k=0, \omega \neq 0$. Besides, they depend on the spatial momentum through $k^{4}$. This implies that when the worrisome terms are written as ratios of polynomials, their numerators are at least proportional to $k^{4}$, which cancels all powers of $k$ from the denominator. This cancellation is in fact guaranteed by the regularity of the propagator $\left\langle h_{i j} h_{k l}\right\rangle$ at $k \rightarrow 0, \omega$-fixed; this, in turn, follows from the regular structure of the kinetic term for $h_{i j}$ in this limit; see (24).

The expressions for the propagators are particularly simple for the choice of the gauge parameters,

$$
\sigma=\frac{1-2 \lambda}{8 \mu(1-\lambda)}, \quad \xi=-\frac{1-2 \lambda}{2(1-\lambda)},
$$

which renders $\mathcal{P}_{1}=\mathcal{P}_{2}=\mathcal{P}_{s}$. Then one obtains

$$
\begin{aligned}
& \left\langle N^{i}(p) N^{j}(-p)\right\rangle=4 \mu \varkappa^{2}\left[\frac{2(1-\lambda)}{1-2 \lambda} \delta_{i j} k^{2}-k_{i} k_{j}\right] \mathcal{P}_{s}(p), \\
& \left\langle h_{i j}(p) h_{k l}(-p)\right\rangle=2 \varkappa^{2}\left[\frac{2 \lambda}{1-2 \lambda} \delta_{i j} \delta_{k l}+\delta_{i k} \delta_{j l}+\delta_{i l} \delta_{j k}\right] \mathcal{P}_{s}(p) .
\end{aligned}
$$

This gauge may be convenient for the actual loop computations in Hořava gravity.

\section{Fadeev-Popov ghosts}

The gauge-fixing procedure must be completed by specifying the action for the Faddeev-Popov ghosts. This is conveniently derived in the Becchi-Rouet-Stora-Tyutin (BRST) formalism $[39,40]$. We follow the presentation of [41]. One introduces an operator $\mathbf{s}$ transforming the metric and the shift,

$\mathbf{s} h_{i j}=\partial_{i} c_{j}+\partial_{j} c_{i}+\partial_{i} c^{k} h_{j k}+\partial_{j} c^{k} h_{i k}+c^{k} \partial_{k} h_{i j}$,

$\mathbf{s} N^{i}=c^{i}-N^{j} \partial_{j} c^{i}+c^{j} \partial_{j} N^{i}$,

where $c^{i}(\tau, \mathbf{x})$ are anticommuting ghost fields. The transformations (29) are nothing but the variations of $h_{i j}$ and $N^{i}$ under infinitesimal diffeomorphisms with the parameters $c^{i}$. Supplementing them by the transformation of the ghosts,

$$
\mathbf{s} c^{i}=c^{j} \partial_{j} c^{i},
$$

it is straightforward to verify that $\mathbf{s}$ is nilpotent, ${ }^{14}$

$$
\mathbf{s}^{2} h_{i j}=\mathbf{s}^{2} N^{i}=\mathbf{s}^{2} c^{i}=0 .
$$

The ghost action is written using the BRST transform of the gauge-fixing function,

$$
S_{g h}=-\frac{1}{\varkappa^{2}} \int \mathrm{d} \tau \mathrm{d}^{2} x \bar{c}_{i}\left(\mathbf{s} F^{i}\right),
$$

where we have introduced the antighost $\bar{c}_{i}$. Explicitly, upon integration by parts we obtain

$$
\begin{aligned}
S_{g h}= & \frac{1}{\varkappa^{2}} \int \mathrm{d} \tau \mathrm{d}^{2} x\left[\dot{\bar{c}}_{i} \dot{c}^{i}+\frac{1}{2 \sigma} \Delta \bar{c}_{i} \Delta c^{i}-\frac{1-2 \lambda+2 \xi(1-\lambda)}{2 \sigma} \partial_{i} \bar{c}_{i} \Delta \partial_{j} c^{j}-\dot{\bar{c}}_{i} \partial_{j} c^{i} N^{j}+\dot{\bar{c}}_{i} c^{j} \partial_{j} N^{i}\right. \\
& \left.-\frac{1}{2 \sigma} \Delta \partial_{j} \bar{c}_{i}\left(\partial_{i} c^{k} h_{j k}+\partial_{j} c^{k} h_{i k}+c^{k} \partial_{k} h_{i j}\right)-\frac{\xi}{2 \sigma} \partial_{i} \partial_{j} \partial_{k} \bar{c}_{i}\left(\partial_{j} c^{l} h_{l k}+\partial_{k} c^{l} h_{j l}+c^{l} \partial_{l} h_{j k}\right)+\frac{\lambda(1+\xi)}{2 \sigma} \Delta \partial_{i} \bar{c}_{i}\left(2 \partial_{k} c^{l} h_{l k}+c^{l} \partial_{l} h\right)\right] .
\end{aligned}
$$

This action is invariant under the anisotropic scaling (1) with the assignment of zero scaling dimension to the (anti-) ghosts,

\footnotetext{
${ }^{13}$ For $\lambda>1$ the second condition implies $\xi<-1$. In this case the operator (22) in the gauge-fixing term is not positive definite. We are not aware of any problems related to this in the perturbation theory. However, it can lead to complications with the nonperturbative definition of the theory [cf. Eq. (42)].

${ }^{14}$ In deriving these identities one uses the graded Leibniz rule, $\mathbf{s} A \cdot B=(\mathbf{s} A) \cdot B+(-1)^{|A|} A \cdot(\mathbf{s} B)$, where $|A|=0(|A|=1)$ for a bosonic (fermionic) field. For example, $\mathbf{s}^{2} c^{i}=\mathbf{s}\left(c^{j} \partial_{j} c^{i}\right)=\left(\mathbf{s} c^{j}\right) \partial_{j} c^{i}-c^{j} \partial_{j}\left(\mathbf{s} c^{i}\right)$.
} 


$$
\left[c^{i}\right]=\left[\bar{c}_{i}\right]=0 .
$$

Note also that the antighost $\bar{c}_{i}$ always appear in the action either with a time derivative or with three spatial derivatives acting on it. From the first line of (33) we obtain the propagator

$$
\left\langle c^{i}(p) \bar{c}_{j}(-p)\right\rangle=\varkappa^{2} \delta_{i j} \mathcal{P}_{1}(p)+\varkappa^{2} \hat{k}_{i} \hat{k}_{j}\left[\mathcal{P}_{2}(p)-\mathcal{P}_{1}(p)\right] .
$$

It is straightforward to check that this propagator is regular. In the gauge (28) it diagonalizes,

$$
\left\langle c^{i}(p) \bar{c}_{j}(-p)\right\rangle=\varkappa^{2} \delta_{i j} \mathcal{P}_{s}(p) .
$$

If we postulate the BRST transformation of the antighost as ${ }^{15}$

$$
\mathbf{s} \bar{c}_{i}=\sigma \mathcal{O}_{i j} F^{j},
$$

the total action composed of the original action, the gaugefixing term (17) and the action for ghosts is BRST invariant,

$$
\begin{aligned}
\mathbf{s}\left[S+S_{g f}+S_{g h}\right] & =\frac{1}{\varkappa^{2}} \int \mathrm{d} \tau \mathrm{d}^{2} x\left[\sigma\left(\mathbf{s} F^{i}\right) \mathcal{O}_{i j} F^{j}-\left(\mathbf{s} \bar{c}_{i}\right)\left(\mathbf{s} F^{i}\right)\right] \\
& =0
\end{aligned}
$$

where we used that the variation of $S$ vanishes as the consequence of gauge invariance, whereas $\mathbf{s}^{2} F^{i}=0$ due to Eqs. (31). In other words, the transformations (29)-(30) and (35) constitute a symmetry of the gauge-fixed action, reflecting the original gauge invariance.

This symmetry explains the following property that at first might appear surprising. The decomposition of the metric and the shift (5) involves four gauge modes: two transverse vectors $u_{i}, v_{i}$ and two longitudinal scalars $B$ and $E$. Adding the gauge-fixing term to the action makes these modes propagating, which naively could give rise to four different pole structures in the propagators of the metric and the shift. Instead, we see only two structures associated with the gauge modes, i.e. $\mathcal{P}_{1}$ and $\mathcal{P}_{2}$. The reason is that the BRST transformation connects the gauge modes to the ghost field which contains only one transverse and one longitudinal component. To see explicitly how this constrains the propagators, consider the linear part of the BRST transformations (29). They form a symmetry of the quadratic action and hence leave the two-point Green's functions invariant. Let us act with $\mathbf{s}$ on the correlators $\left\langle h_{i j} \bar{c}_{k}\right\rangle$ and $\left\langle N^{i} \bar{c}_{k}\right\rangle$ which trivially vanish. We obtain

\footnotetext{
${ }^{15}$ With this definition the second BRST variation of the antighost is nonzero, $\mathbf{s}^{2} \bar{c}_{i}=\sigma \mathcal{O}_{i j} \mathbf{s} F^{j} \neq 0$. It is possible to modify the formalism in such a way that $\mathbf{s}^{2}$ will annihilate all fields, including the antighost, at the expense of introducing an additional auxiliary variable. We prefer to avoid this complication which is irrelevant for our purposes.
}

$$
\begin{aligned}
0=\mathbf{s}\left\langle h_{i j} \bar{c}_{k}\right\rangle & =\left\langle\left(\partial_{i} c_{j}+\partial_{j} c_{i}\right) \bar{c}_{k}\right\rangle+\sigma\left\langle h_{i j} \mathcal{O}_{k l} F^{l}\right\rangle \\
& =\left\langle\left(\partial_{i} c_{j}+\partial_{j} c_{i}\right) \bar{c}_{k}\right\rangle+\frac{1}{2}\left\langle h_{i j}\left(\partial_{l} h_{k l}-\lambda \partial_{k} h\right)\right\rangle,
\end{aligned}
$$

where passing to the second line we substituted the explicit form (23) of the gauge-fixing function and used that the correlator $\left\langle h_{i j} N^{k}\right\rangle$ vanishes. Similarly

$$
0=\mathbf{s}\left\langle N^{i} \bar{c}_{j}\right\rangle=\left\langle\dot{c}^{i} \bar{c}_{j}\right\rangle+\sigma \mathcal{O}_{j k}\left\langle N^{i} \dot{N}^{k}\right\rangle .
$$

These relations imply, in particular, that the poles of the gauge modes must coincide with the poles of the ghost propagator and thus there can be at most two gaugedependent poles. It is straightforward to verify that the propagators (25), (34) satisfy the above relations.

\section{B. Theory in three spatial dimensions}

The analysis of the previous section can be easily generalized to projectable Hořava gravity in spacetime of arbitrary dimension $(d+1)$. We work out explicitly the case $d=3$, the lowest dimensionality admitting propagating TT mode. We do not repeat the details of the derivation, highlighting only the difference from the $d=2$ case. For the sake of clarity, we keep only marginal terms in the potential (4) omitting the relevant deformations. The latter do not affect the UV properties of the theory that are of interest to us.

The gauge-fixing Lagrangian is still given by the expression (17). However, now the scaling dimension of $F^{i}$, which coincides with the dimension of $\dot{N}^{i}$, is 5 . Therefore, for the gauge-fixing term to be scale invariant, the operator $\mathcal{O}_{i j}$ must have dimension ${ }^{16}-4$. Its general form is

$$
\mathcal{O}_{i j}=\Delta^{-1}\left[\delta_{i j} \Delta+\xi \partial_{i} \partial_{j}\right]^{-1} .
$$

Substituting this into the first line of (23) we obtain the explicit expression for the gauge-fixing function,

$F^{i}=\dot{N}^{i}+\frac{1}{2 \sigma} \Delta^{2} \partial_{k} h_{i k}-\frac{\lambda(1+\xi)}{2 \sigma} \Delta^{2} \partial_{i} h+\frac{\xi}{2 \sigma} \Delta \partial_{i} \partial_{j} \partial_{k} h_{j k}$.

As in $d=2$, this choice of the gauge-fixing function eliminates the crossterms mixing the shift and the metric in the quadratic action. One combines $\mathcal{L}_{g f}$ with the quadratic Lagrangian (8), sets to zero the coefficients in front of the relevant deformations, $\eta=\mu_{1}=\mu_{2}=0$, and flips the sign of $\nu_{4,5}$ in consequence of the Wick rotation. Then, a straightforward calculation yields the nonzero propagators,

$$
\begin{aligned}
\left\langle N^{i}(p) N^{j}(-p)\right\rangle= & \frac{\varkappa^{2} k^{2}}{\sigma}\left(k^{2} \delta_{i j}-k_{i} k_{j}\right) \mathcal{P}_{1}(p) \\
& +\frac{\varkappa^{2}(1+\xi) k^{2}}{\sigma} k_{i} k_{j} \mathcal{P}_{2}(p),
\end{aligned}
$$

\footnotetext{
${ }^{16}$ Recall that for $d=3$ the scaling dimension of the spacetime measure is $\left[\mathrm{d} \tau \mathrm{d}^{3} x\right]=-6$.
} 


$$
\begin{aligned}
\left\langle h_{i j}(p) h_{k l}(-p)\right\rangle= & 2 \varkappa^{2}\left(\delta_{i k} \delta_{j l}+\delta_{i l} \delta_{j k}\right) \mathcal{P}_{t t}(p)-2 \varkappa^{2} \delta_{i j} \delta_{k l}\left[\mathcal{P}_{t t}(p)-\frac{1-\lambda}{1-3 \lambda} \mathcal{P}_{s}(p)\right] \\
& -2 \varkappa^{2}\left(\delta_{i k} \hat{k}_{j} \hat{k}_{l}+\delta_{i l} \hat{k}_{j} \hat{k}_{k}+\delta_{j k} \hat{k}_{i} \hat{k}_{l}+\delta_{j l} \hat{k}_{i} \hat{k}_{k}\right)\left[\mathcal{P}_{t t}(p)-\mathcal{P}_{1}(p)\right] \\
& +2 \varkappa^{2}\left(\delta_{i j} \hat{k}_{k} \hat{k}_{l}+\hat{k}_{i} \hat{k}_{j} \delta_{k l}\right)\left[\mathcal{P}_{t t}(p)-\mathcal{P}_{s}(p)\right] \\
& +2 \varkappa^{2} \hat{k}_{i} \hat{k}_{j} \hat{k}_{k} \hat{k}_{l}\left[\mathcal{P}_{t t}(p)+\frac{1-3 \lambda}{1-\lambda} \mathcal{P}_{s}(p)-4 \mathcal{P}_{1}(p)+\frac{2 \mathcal{P}_{2}(p)}{1-\lambda}\right]
\end{aligned}
$$

where now the pole structures are

$$
\begin{aligned}
& \mathcal{P}_{t t}=\frac{1}{\omega^{2}+\nu_{4} k^{6}}, \\
& \mathcal{P}_{s}=\left[\omega^{2}+\frac{\left(8 \nu_{4}+3 \nu_{5}\right)(1-\lambda)}{1-3 \lambda} k^{6}\right]^{-1}, \\
& \mathcal{P}_{1}=\left[\omega^{2}+\frac{k^{6}}{2 \sigma}\right]^{-1}, \\
& \mathcal{P}_{2}=\left[\omega^{2}+\frac{(1-\lambda)(1+\xi)}{\sigma} k^{6}\right]^{-1} .
\end{aligned}
$$

The first two structures correspond to the physical TT and scalar modes, cf. Eqs. (9), whereas the other two are gauge dependent. Note that the latter coincide with the expressions (26) up to the substitution $k^{4} \mapsto k^{6}$. By inspection one finds that the propagators (36) satisfy the regularity conditions (19).

The BRST transformations have the same form as before, Eqs. (29)-(30) and (35). This also applies to the ghost action which is given by (32), up to replacement of the integration measure $\mathrm{d} \tau \mathrm{d}^{2} x \mapsto \mathrm{d} \tau \mathrm{d}^{3} x$. We write down explicitly only the quadratic part,

$$
\begin{aligned}
S_{g h}= & \frac{1}{\varkappa^{2}} \int \mathrm{d} \tau \mathrm{d}^{3} x\left[\dot{\bar{c}}_{i} \dot{c}^{i}-\frac{1}{2 \sigma} \bar{c}_{i} \Delta^{3} c^{i}\right. \\
& \left.+\frac{1-2 \lambda+2 \xi(1-\lambda)}{2 \sigma} \partial_{i} \bar{c}_{i} \Delta^{2} \partial_{j} c^{j}+\cdots\right],
\end{aligned}
$$

where dots stand for cubic terms describing interactions of ghosts with $N^{i}$ and $h_{i j}$. From this action one reads off the expression for the ghost propagator which turns out to be the same as (34), but with $\mathcal{P}_{1,2}$ given by Eqs. (37c)-(37d).

One can use the freedom in the choice of the gauge parameters $\sigma$ and $\xi$ to simplify the expressions (36). However, unlike the case $d=2$, it is generically impossible to make all propagators proportional to each other. This is, of course, due to the presence of two distinct physical excitations in the theory-TT and scalar gravitons - that in general have different dispersion relations.

\section{COUNTERTERMS}

In this section we argue that existence of $\sigma \xi$-gauges where all propagators are regular implies renormalizability. We carry out the derivation for the case $d=2$; the generalization to higher dimensions is straightforward.

\section{A. Degree of divergence}

We work with the total action

$$
S_{\mathrm{tot}}=S+S_{g f}+S_{g h},
$$

where $S$ is given by (3) with the potential (10), the gaugefixing term $S_{g f}$ corresponds to the Lagrangian (17) and the ghost action $S_{g h}$ has the form (32). Consider a general diagram appearing in the perturbative expansion based on this action. One introduces the notations

(i) $P_{h h}$-number of $\left\langle h_{i j} h_{k l}\right\rangle$ propagators,

(ii) $P_{N N}$-number of $\left\langle N^{i} N^{j}\right\rangle$ propagators,

(iii) $P_{c c}$-number of the ghost propagators,

(iv) $V_{[h]}$-number of vertices involving only the $h_{i j}$-fields,

(v) $V_{[h] N}$-number of vertices with an arbitrary number of $h$-legs and a single $N$-leg,

(vi) $V_{[h] N N}$-number of vertices with an arbitrary number of $h$-legs and two $N$-legs,

(vii) $V_{h c c}$-number of vertices describing interaction of $h_{i j}$ with the ghosts,

(viii) $V_{N c c}$-number of vertices describing interaction of $N^{i}$ with the ghosts,

(ix) $L$-number of loops, i.e. number of independent loop integrals,

(x) $l_{N}$-number of external $N$-legs,

(xi) $T$-number of time derivatives acting on external legs,

(xii) $X$-number of spatial derivatives acting on external legs.

These quantities obey two relations:

$$
\begin{aligned}
L= & P_{h h}+P_{N N}+P_{c c}-V_{[h]}-V_{[h] N}-V_{[h] N N}-V_{h c c} \\
& -V_{N c c}+1 \\
l_{N}= & V_{[h] N}+V_{N c c}+2 V_{[h] N N}-2 P_{N N} .
\end{aligned}
$$

The first relation follows from the standard reasoning that out of $\left(P_{h h}+P_{N N}+P_{c c}\right)$ original integrals over 
frequencies and momenta $\left(V_{[h]}+V_{[h] N}+V_{[h] N N}+V_{h c c}+\right.$ $\left.V_{N c c}-1\right)$ are removed by the $\delta$-functions at the vertices (one $\delta$-function remains as an overall factor multiplying the whole diagram). The second relation is obtained by counting the $N$-legs. Indeed, each vertex of the type $V_{[h] N}$ or $V_{N c c}$ brings one $N$-leg, whereas the vertex $V_{[h] N N}$ brings two; every $\left\langle N^{i} N^{j}\right\rangle$-propagator absorbs two legs; the remaining $N$ legs are external.

Next we introduce the superficial degree of divergence $D_{\text {div }}$ of the diagram. This is defined as the scaling power of the diagram under the simultaneous rescaling of all loop momenta and frequencies,

$$
\mathbf{k}^{(l)} \mapsto b \mathbf{k}^{(l)}, \quad \omega^{(l)} \mapsto b^{2} \omega^{(l)},
$$

in the limit $b \rightarrow \infty$. By inspection of the expressions for the propagators and vertices one obtains

$$
\begin{aligned}
D_{\text {div }}= & 4 L-4 P_{h h}-2 P_{N N}-4 P_{c c}+4 V_{[h]}+3 V_{[h] N} \\
& +2 V_{[h] N N}+4 V_{h c c}+3 V_{N c c}-2 T-X .
\end{aligned}
$$

Using (38) this reduces to ${ }^{17}$

$$
D_{\text {div }}=4-2 T-X-l_{N} \text {. }
$$

Let us focus on the diagrams with external $h$-legs only. We see that $D_{\text {div }}$ is negative for diagrams with more than two time or four space derivatives on external legs. Assuming that $D_{\text {div }}<0$ implies convergence of a diagram, one concludes that only diagrams with at most two time and four space derivatives on the external lines must be renormalized. These diagrams can be Taylor expanded ${ }^{18}$ in the external frequencies and momenta, with the successive terms in the series having lower and lower degrees of divergence. Starting from a certain order the coefficients in the Taylor expansion become finite, so that only a few first terms in the series will require subtraction. The corresponding counterterms are polynomial in external frequencies and momenta and hence local in position space. Again, they have no more than two time or four space derivatives acting on the metric $h_{i j}$. In other words, their scaling dimension is less than or equal to four. If we further assume that the divergent parts of the diagrams respect the foliation-preserving diffeomorphisms, it follows that the counterterms must have the same form as the terms already present in the action (3), (10). This amounts to renormalizability.

The above argument contains two important assumptions that we now scrutinize. First, a generic diagram will contain

\footnotetext{
${ }^{17}$ The generalization of this formula for $(d+1)$-dimensional Hořava gravity is $D_{\text {div }}=2 d-d \cdot T-X-(d-1) l_{N}$.

${ }^{18} \mathrm{We}$ assume that the UV divergences have been appropriately regulated, e.g. by analytically continuing in the dimensionalities of time and space [24], and that possible IR divergences have been removed by introducing an IR cutoff.
}

subdivergences and thus can diverge despite $D_{\text {div }}<0$. Fortunately, as shown in [24], the combinatorics of the subtraction procedure in nonrelativistic theories work essentially in the same way as in the relativistic case, and subdivergences are subtracted by counterterms introduced at the previous orders of the loop expansion.

Second, even in the absence of subdivergences, the convergence of a diagram with $D_{\text {div }}<0$ is not trivial. Indeed, consider the integral

$$
\int \mathrm{d} \omega^{(1)} \mathrm{d}^{2} k^{(1)} \int \prod_{l=2}^{L}\left[\mathrm{~d} \omega^{(l)} \mathrm{d}^{2} k^{(l)}\right] f(\{\omega\},\{\mathbf{k}\})
$$

where we singled out the first loop momentum and suppressed the dependence on external momenta. Assume for simplicity that $f$ is a scalar function (in general it can carry tensor indices corresponding to the external legs of the diagram). Because subdivergences are absent, the inner integral converges and gives a function $\tilde{f}\left(\omega^{(1)}, k^{(1)}\right)$ which for $\mathbf{k}^{(1)} \mapsto b \mathbf{k}^{(1)}, \omega^{(1)} \mapsto b^{2} \omega^{(1)}$ scales as $b^{D_{\text {div }}-4}$. However, the latter can have the form

$$
\begin{gathered}
\tilde{f} \sim\left(\omega^{(1)}\right)^{-1+n}\left(k^{(1)}\right)^{D_{\text {div }}-2-2 n} \\
\text { or }\left(\omega^{(1)}\right)^{-1-n}\left(k^{(1)}\right)^{D_{\text {div }}-2+2 n}, \\
n>0,
\end{gathered}
$$

and the integral over frequency (momentum) will diverge, despite the fact that the $k$-integral ( $\omega$-integral) is finite. These are precisely the spurious divergences that arise if the propagators contain irregular contributions discussed in Sec. III. Note that this problem is absent in Lorentz invariant theories, where the function $\tilde{f}$ can depend only on $\left(\omega^{(1)}\right)^{2}+$ $\left(k^{(1)}\right)^{2}$. In Appendix B we prove that spurious divergences (39) do not appear if all propagators have the regular form (19). In that case $D_{\text {div }}<0$ indeed implies convergence of the diagram.

Finally, we must discuss the gauge invariance of the counterterms. In the perturbative expansion around flat spacetime, which we have been considering so far, gauge invariance is actually not preserved. One way to proceed would be to exploit the BRST symmetry of the gauge-fixed action to constrain the structure of counterterms, similar to the analysis of [2]. This approach would require considering divergent diagrams with external ghost lines (as well as diagrams with external $N$-legs) and working out their relation to the diagrams renormalizing the vertices containing only the metric. This should be done order by order in perturbation theory, and the analysis is very cumbersome. Instead, we adopt the method of background effective action ${ }^{19}$ where the invariance with respect to the (background) gauge transformations is manifest.

\footnotetext{
${ }^{19}$ See [42-44] for a pedagogical introduction.
} 


\section{B. Background-covariant formulation}

One decomposes the total metric and shift into background $\bar{\gamma}_{i j}, \bar{N}^{i}$ and fluctuations $h_{i j}, n^{i}$ :

$$
\gamma_{i j}=\bar{\gamma}_{i j}+h_{i j}, \quad N^{i}=\bar{N}^{i}+n^{i} .
$$

We still have to fix the gauge for the fluctuations. However, one can do it in the way that explicitly preserves the invariance with respect to diffeomorphisms acting on the background. This is easily achieved by covariantizing all formulas of the previous sections. Instead of (22)-(23) we write

$$
F^{i}=\bar{D}_{t} n^{i}+\frac{1}{2 \sigma}\left(\mathcal{O}^{-1}\right)^{i j} \bar{\nabla}_{k} h_{j}^{k}-\frac{\lambda}{2 \sigma}\left(\mathcal{O}^{-1}\right)^{i j} \bar{\nabla}_{j} h,
$$

where

$$
\bar{D}_{t} n^{i}=\dot{n}^{i}-\bar{N}^{k} \bar{\nabla}_{k} n^{i}+\bar{\nabla}_{k} \bar{N}^{i} n^{k}
$$

is the covariant time derivative and

$$
\mathcal{O}_{i j}=-\left[\bar{\Delta} \bar{\gamma}^{i j}+\xi \bar{\nabla}^{i} \bar{\nabla}^{j}\right]^{-1}
$$

The covariant derivatives $\bar{\nabla}_{i}$ are defined using the Christoffel connection constructed from $\bar{\gamma}_{i j}$; all indices are raised and lowered with $\bar{\gamma}^{i j}, \bar{\gamma}_{i j}$, and $h=h_{i j} \bar{\gamma}^{i j}$. The gauge-fixing action is still given by the Lagrangian (17) that must be integrated over the spacetime with the covariant measure $\int d \tau d^{2} x \sqrt{\bar{\gamma}}$. Similarly, the ghost action has the form

$$
S_{g h}=-\frac{1}{\varkappa^{2}} \int \mathrm{d} \tau \mathrm{d}^{2} x \sqrt{\bar{\gamma}} \bar{c}_{i}\left(\mathbf{s} F^{i}\right),
$$

where BRST transformations of the fields are defined by promoting all derivatives in (29)-(30) and (35) to be background-covariant. For example, for the shift we have,

$$
\mathbf{s} n^{i}=\bar{D}_{t} c^{i}-n^{j} \bar{\nabla}_{j} c^{i}+c^{j} \bar{\nabla}_{j} n^{i} .
$$

It is straightforward, even if somewhat tedious, to verify that the action of the BRST operator on $h_{i j}, n^{i}$ and $c^{i}$ is still nilpotent.

The idea of the background-field method is to take the path integral over the fluctuations and obtain an effective action for the background fields. The latter will be automatically invariant with respect to background gauge transformations. ${ }^{20}$ In particular, this holds for its divergent part that requires renormalization. In other words, in the background-covariant formulation the counterterms are guaranteed to be gauge invariant.

\footnotetext{
${ }^{20} \mathrm{We}$ assume dimensional regularization, which preserves gauge invariance.
}

One should be worried at this point that the gauge-fixing Lagrangian depends on the background fields in a nonlocal manner which can compromise the locality of the counterterms. To resolve this issue, we observe that the nonlocal operator $\mathcal{O}_{i j}$ actually cancels everywhere in the gaugefixing action, except the kinetic term for the shift,

$$
S_{n, \text { kin }}=\int \mathrm{d} \tau \mathrm{d}^{2} x \sqrt{\bar{\gamma}} \frac{\sigma}{2 \varkappa^{2}} \bar{D}_{t} n^{i} \mathcal{O}_{i j} \bar{D}_{t} n^{j} .
$$

The latter is cast in the local form by introducing an auxiliary field $\pi_{i}$,

$S_{n, \text { kin }}^{\prime}=\frac{1}{\varkappa^{2}} \int \mathrm{d} \tau \mathrm{d}^{2} x \sqrt{\bar{\gamma}}\left[\frac{1}{2 \sigma} \pi_{i}\left(\mathcal{O}^{-1}\right)^{i j} \pi_{j}-i \pi_{i} \bar{D}_{t} n^{i}\right]$.

Taking the Gaussian path integral over $\pi_{i}$ reproduces (40). Note that we have introduced an imaginary coefficient in front of the second term in (41) in order to preserve the positivity of the quadratic term. ${ }^{21}$ This is not problematic: the imaginary part of (41) is odd when $\pi_{i}$ changes sign and hence the effective action is real as it is obtained by integrating over all values of $\pi_{i}$. Besides, we notice that $\pi_{i}$ enters in the action as a canonically conjugate momentum for the shift perturbations $n^{i}$. From this perspective, the presence of an imaginary part in (41) is not surprising. Indeed, such an imaginary part associated with the canonical form appears even in ordinary mechanics when the Euclidean action is written in terms of canonical variables.

It is instructive to work out how the introduction of $\pi_{i}$ affects the measure in the path integral. Let us take a step backward and recall that the gauge-fixing Lagrangian (17) arises as a result of smearing the gauge-fixing condition $F^{i}=f^{i}$ with the weighting functional

$$
\sqrt{\operatorname{Det} \mathcal{O}_{i j}} \int\left[\mathrm{d} f^{i}\right] \exp \left[-\int \mathrm{d} \tau \mathrm{d}^{2} x \sqrt{\bar{\gamma}} \frac{\sigma}{2 \varkappa^{2}} f^{i} \mathcal{O}_{i j} f^{j}\right]
$$

inserted in the partition function of the theory. Notice the square root of the functional determinant of the operator $\mathcal{O}_{i j}$ in the prefactor which ensures the correct normalization. Thus, before introducing $\pi_{i}$ the partition function has the form

$$
Z=\sqrt{\operatorname{Det} \mathcal{O}_{i j}} \int\left[\mathrm{d} n^{j} \mathrm{~d} h_{k l} \mathrm{~d} c^{m} \mathrm{~d} \bar{c}_{n}\right] \exp \left[-\left(S_{n, \text { kin }}+\cdots\right)\right],
$$

where the ellipsis stands for the local contributions in the action. The introduction of $\pi_{i}$ not only makes the action local, but also absorbs the determinant from the prefactor. This follows from the relations

\footnotetext{
${ }^{21}$ Strictly speaking, this argument applies in the case $\lambda<1 / 2$ when the operator $\mathcal{O}_{i j}$, and hence $\left(\mathcal{O}^{-1}\right)^{i j}$, is positive definite. For $\lambda>1$ the positivity cannot be ensured, which, however, does not affect the perturbative considerations; see footnote 13 .
} 


$$
\begin{aligned}
\mathrm{e}^{-S_{n, k i n}\left[n^{i}\right]} & =\sqrt{\operatorname{Det}\left(\mathcal{O}^{-1}\right)^{i j}} \int\left[\mathrm{d} \pi_{j}\right] \mathrm{e}^{-S_{n, k i n}^{\prime}\left[\pi_{j}, n^{i}\right]}, \\
\sqrt{\operatorname{Det} \mathcal{O}_{i j}} \sqrt{\operatorname{Det}\left(\mathcal{O}^{-1}\right)^{i j}} & =1 .
\end{aligned}
$$

Thus, we arrive to the final expression for the partition function,

$$
Z=\int\left[\mathrm{d} \pi_{i} \mathrm{~d} n^{j} \mathrm{~d} h_{k l} \mathrm{~d} c^{m} \mathrm{~d} \bar{c}_{n}\right] \exp \left[-\left(S_{n, \text { kin }}^{\prime}+\cdots\right)\right]
$$

Curiously, the introduction of $\pi_{i}$ makes the integration measure in the path integral flat, which further supports the identification of $\pi_{i}$ as the canonically conjugate momentum to $n^{i}$.

Last but not least, we have to check that the introduction of $\pi_{i}$ does not spoil the regular structure of the propagators. It is sufficient to perform this analysis for the flat background, $\bar{\gamma}_{i j}=\delta_{i j}, \bar{N}^{i}=0$, as locally any background can be brought to this form by a coordinate choice and our question deals with the local properties of the propagators. From the quadratic Lagrangian in the $\pi n$-sector,

$$
\begin{aligned}
\mathcal{L}_{2, \pi n}^{d=2}= & \frac{1}{2 \varkappa^{2}}\left[-\frac{1}{\sigma} \pi_{i}\left(\Delta \delta_{i j}+\xi \partial_{i} \partial_{j}\right) \pi_{j}-2 i \pi_{k} \dot{n}^{k}+\frac{\left(\partial_{i} n^{j}\right)^{2}}{2}\right. \\
& \left.+\left(\frac{1}{2}-\lambda\right)\left(\partial_{i} n^{i}\right)^{2}\right]
\end{aligned}
$$

we find that the $\left\langle n^{i} n^{j}\right\rangle$ propagator is not modified and is given by (25a), whereas

$$
\begin{aligned}
\left\langle\pi_{i}(p) n^{j}(-p)\right\rangle= & \varkappa^{2} \omega \delta_{i j} \mathcal{P}_{1}(p)+\varkappa^{2} \omega \hat{k}_{i} \hat{k}_{j}\left[\mathcal{P}_{2}(p)-\mathcal{P}_{1}(p)\right], \\
\left\langle\pi_{i}(p) \pi_{j}(-p)\right\rangle= & \frac{\varkappa^{2}}{2}\left(k^{2} \delta_{i j}-k_{i} k_{j}\right) \mathcal{P}_{1}(p) \\
& +\varkappa^{2}(1-\lambda) k_{i} k_{j} \mathcal{P}_{2}(p)
\end{aligned}
$$

These are compatible with the regular form (19) for the scaling dimension ${ }^{22}\left[\pi_{i}\right]=1$. As a consequence, the reasoning of Sec. VA goes through essentially unchanged with the field $\pi_{i}$ taken into consideration.

To sum up, we have formulated a manifestly background-covariant gauge-fixing procedure. Combined with the results of Sec. VA about the scaling dimension of possible divergences, it implies that at one loop counterterms in the effective action have the same structure as the terms in the bare Lagrangian. There are strong arguments that this also holds at higher loops [45-48] implying that the theory is renormalizable.

\footnotetext{
${ }^{22}$ The fact that the scaling dimension of $\pi_{i}$ is equal to 1 follows from the way it enters into the action multiplied by the time derivative of the shift. This applies to Hořava gravity in any number of spacetime dimensions.
}

\section{NONPROJECTABLE MODEL}

We now consider the nonprojectable Hořava gravity. Again, for illustration of the general situation we take the model in $(2+1)$ dimensions [49] which is technically much simpler than its $(3+1)$-dimensional counterpart. Upon using integration by parts and the fact that in two dimensions the Riemann tensor is expressed in terms of the scalar curvature, one finds that the potential contains ten inequivalent terms,

$$
\begin{aligned}
\mathcal{V}= & 2 \Lambda-\eta R-\alpha a_{i} a^{i}+\mu R^{2}+\rho_{1} \Delta R+\rho_{2} R a_{i} a^{i} \\
& +\rho_{3}\left(a_{i} a^{i}\right)^{2}+\rho_{4} a_{i} a^{i} \nabla_{j} a^{j}+\rho_{5}\left(\nabla_{j} a^{j}\right)^{2}+\rho_{6} \nabla_{i} a_{j} \nabla^{i} a^{j},
\end{aligned}
$$

where

$$
a_{i}=\frac{\partial_{i} N}{N}
$$

is the combination of the lapse and its derivative which is invariant under the reparametrizations of time; see Eqs. (2). Expanding around flat spacetime, ${ }^{23}$ one obtains the quadratic action

$$
\begin{aligned}
S_{2}^{d=2, n p}= & \frac{1}{2 \varkappa^{2}} \int \mathrm{d} t \mathrm{~d}^{2} x\left[-\frac{1}{2}\left(\dot{v}_{i}-u_{i}\right) \Delta\left(\dot{v}_{i}-u_{i}\right)+\frac{\dot{\psi}^{2}}{4}\right. \\
& +\frac{1}{4}(\dot{E}-2 \Delta B)^{2}-\frac{\lambda}{4}(\dot{\psi}+\dot{E}-2 \Delta B)^{2}-\eta \phi \Delta \psi \\
& \left.-\alpha \phi \Delta \phi-\mu(\Delta \psi)^{2}+\rho_{1} \phi \Delta^{2} \psi-\left(\rho_{5}+\rho_{6}\right) \phi \Delta^{2} \phi\right],
\end{aligned}
$$

where we used the helicity decomposition (5) and introduced the fluctuation of the lapse $\phi \equiv N-1$. This action propagates a single scalar degree of freedom with the dispersion relation,

$$
\begin{aligned}
\omega^{2}= & \left(\frac{1-\lambda}{1-2 \lambda}\right) \\
& \times \frac{\eta^{2} k^{2}+\left(4 \alpha \mu+2 \eta \rho_{1}\right) k^{4}+\left(\rho_{1}^{2}-4 \mu\left(\rho_{5}+\rho_{6}\right)\right) k^{6}}{\alpha-\left(\rho_{5}+\rho_{6}\right) k^{2}} .
\end{aligned}
$$

In contrast to the projectable case, this dispersion relation is linear at low $k$,

$$
\omega^{2}=\left(\frac{1-\lambda}{1-2 \lambda}\right) \frac{\eta^{2}}{\alpha} k^{2}
$$

At large momenta it respects the anisotropic scaling (1),

$$
\omega^{2}=\frac{1-\lambda}{1-2 \lambda}\left(4 \mu-\frac{\rho_{1}^{2}}{\rho_{5}+\rho_{6}}\right) k^{4} .
$$

\footnotetext{
${ }^{23}$ For the flat spacetime to be a solution, we assume that the cosmological constant $\Lambda$ is tuned to zero.
} 
The mode has positive energy and is stable at all momenta for an appropriate choice of parameters. In particular, the following necessary conditions must be satisfied: $\varkappa^{2}>0, \lambda<$ $1 / 2$ or $\lambda>1, \alpha>0,\left(\rho_{5}+\rho_{6}\right)<0$ and $4 \mu>\rho_{1}^{2} /\left(\rho_{5}+\rho_{6}\right)$.

Let us focus on the UV behavior of the model. Accordingly, we retain only marginal terms in the action which amounts to setting $\eta=\alpha=0$. Next, one performs the Wick rotation and introduces the gauge-fixing term (17) with the gauge-fixing function (23) and $\mathcal{O}_{i j}$ defined in (22). After a somewhat lengthy, but straightforward calculation one finds that the propagators of the shift and the metric have the form (25), where $\mathcal{P}_{1}, \mathcal{P}_{2}$ are still given by (26), while

$$
\mathcal{P}_{s}(p)=\left[\omega^{2}+\frac{1-\lambda}{1-2 \lambda}\left(4 \mu-\frac{\rho_{1}^{2}}{\rho_{5}+\rho_{6}}\right) k^{4}\right]^{-1} .
$$

Other nonvanishing propagators are

$$
\begin{aligned}
\langle\phi(p) \phi(-p)\rangle= & \frac{\varkappa^{2}(1-\lambda) \rho_{1}^{2}}{(1-2 \lambda)\left(\rho_{5}+\rho_{6}\right)^{2}} \mathcal{P}_{s}(p)+\frac{\varkappa^{2}}{\left(\rho_{5}+\rho_{6}\right) k^{4}} \\
\left\langle\phi(p) h_{i j}(-p)\right\rangle= & \frac{2 \varkappa^{2}(1-\lambda) \rho_{1}}{(1-2 \lambda)\left(\rho_{5}+\rho_{6}\right)} \delta_{i j} \mathcal{P}_{s}(p) \\
& -\frac{2 \varkappa^{2} \rho_{1} k_{i} k_{j}}{\left(\rho_{5}+\rho_{6}\right) k^{2}} \mathcal{P}_{s}(p) .
\end{aligned}
$$

Clearly, the last terms in (43a)-(43b) violate the regularity condition. Though these contributions have been derived within a particular family of gauges, we believe they cannot be removed by any gauge choice. They correspond to the instantaneous interaction present in the theory $[12,26]$. We conclude that the correlators of the lapse contain genuinely nonlocal terms whose contributions to the loop diagrams must be carefully analyzed to establish or disprove the renormalizability of the theory.

\section{CONCLUSIONS}

In this paper we have demonstrated renormalizability of the projectable version of Hořava gravity. Though for concreteness we focused on the models in $d=2$ and $d=$ 3 spatial dimensions, our analysis is completely general and applies to Hořava gravity in any dimensionality. Thus, for $d \geq 3$ projectable Hořava gravity presents the first example of unitary renormalizable quantum field theory of gravitation with dynamical transverse-traceless excitations (gravitons). The key element of our argument is the choice of gauge fixing which ensures the right scaling properties of the propagators and their uniform falloff at large frequencies and momenta. The latter property is essential to guarantee locality of the singularities of the propagators in position space. An unusual feature of our approach is that it involves a gauge-fixing term which is nonlocal in space.
We showed how this nonlocality can be resolved by introduction of an auxiliary field resulting in a local gauge-fixed action.

We restricted the analysis to pure gravity theories. We now argue that the renormalizability is preserved upon inclusion of matter obeying the Lifshitz scaling (1) in the UV. Indeed, the derivation of Sec. V relies only on the scaling properties of the propagators and vertices, regular form of the propagators, and invariance under foliationpreserving diffeomorphisms. These properties are clearly satisfied by Lifshitz scalars and fermions, and in general, by any Lifshitz matter with only global internal symmetries. For gauge theories one should use an appropriate gauge fixing that leads to regular propagators. It turns out that this gauge fixing is nonlocal, in complete analogy with what we have found for gravity. For example, for a gauge field $\left(\mathcal{A}_{0}, \mathcal{A}_{i}\right)$ in $d=2$ with the quadratic Euclidean Lagrangian,

$$
\begin{aligned}
\mathcal{L}_{A} & =\frac{1}{2} \mathcal{F}_{0 i} \mathcal{F}^{0 i}-\frac{\mu_{A}}{4} \mathcal{F}_{i j} \Delta \mathcal{F}^{i j}, \\
\mathcal{F}_{0 i} & =\dot{\mathcal{A}}_{i}-\partial_{i} \mathcal{A}_{0}, \\
\mathcal{F}_{i j} & =\partial_{i} \mathcal{A}_{j}-\partial_{j} \mathcal{A}_{i},
\end{aligned}
$$

the suitable gauge-fixing term is

$$
\mathcal{L}_{A, g f}=-\frac{\sigma_{A}}{2}\left(\dot{\mathcal{A}}_{0}+\frac{1}{\sigma_{A}} \Delta \partial_{i} \mathcal{A}_{i}\right) \frac{1}{\Delta}\left(\dot{\mathcal{A}}_{0}+\frac{1}{\sigma_{A}} \Delta \partial_{j} \mathcal{A}_{j}\right) .
$$

It is chosen in such a way to cancel the crossterms mixing $\mathcal{A}_{0}$ and $\mathcal{A}_{i}$. For $\sigma_{A}=1 / \mu_{A}$ the quadratic action diagonalizes completely and the propagators are particularly simple,

$$
\left\langle\mathcal{A}_{0} \mathcal{A}_{0}\right\rangle=\frac{\mu_{A} k^{2}}{\omega^{2}+\mu_{A} k^{4}}, \quad\left\langle\mathcal{A}_{i} \mathcal{A}_{j}\right\rangle=\frac{\delta_{i j}}{\omega^{2}+\mu_{A} k^{4}} .
$$

These are regular and correspond to the dimensions $\left[\mathcal{A}_{0}\right]=1,\left[\mathcal{A}_{i}\right]=0$ dictated by the anisotropic scale invariance of the Lagrangian (44). Furthermore, the nonlocality of the gauge-fixing term gets resolved by introducing a canonically conjugate momentum for $\mathcal{A}_{0}$, as done in Sec. V B for gravity. We conclude that the projectable Hořava gravity can be coupled also to gauge fields with Lifshitz scaling without spoiling renormalizability.

Given renormalizability, the next obvious question is the renormalization group (RG) behavior of the coupling constants. Only when the RG flow has a weakly coupled UV fixed point can the theory be considered UV complete. If instead it turns out that the running couplings develop a Landau pole, the theory will still require embedding into a broader framework. Our results imply that the running of the couplings is logarithmic, and thus the need for such embedding, if any, will be postponed to exponentially high energy scales. 
Another issue that acquires new importance in view of our findings is the fate of the instability at low momenta present in $(3+1)$-dimensional projectable Hořava gravity. It would be interesting to understand whether this instability can be cut off by nonlinear terms in the Lagrangian and, if it can, determine the structure of the ground state. The fact that the instability is developed by the modes with nonzero spatial momenta suggests that the putative ground state will break translational invariance.

For the nonprojectable Hořava gravity our gauge-fixing procedure is not sufficient to establish renormalizability. We have found that it still leaves certain contributions in the propagators of the lapse that do not fall off with frequency. These contributions cannot be removed by any gauge choice and are related to the physical instantaneous interaction present in the theory. In position space they lead to singularities in the propagators that are nonlocal in space. It will be crucial to work out the implications of this nonlocality for the perturbative expansion in order to establish (or disprove) renormalizability of the theory. We leave this study for the future.

\section{ACKNOWLEDGMENTS}

We are indebted to Enrique Álvarez, Dmitry Levkov, Valery Rubakov and Arkady Vainshtein for stimulating discussions. We thank Nikolai Krasnikov and Mikhail Shaposhnikov for encouraging interest. A. B. is grateful for the hospitality and support of Theory Division, CERN, and the Pacific Institute of Theoretical Physics, University of British Columbia. The work of A. B. was also supported by the RFBR Grant No. 14-02-01173 and by the Tomsk State University Competitiveness Improvement Program. M. H-.V. wishes to thank the Institut de Théorie des Phénomènes Physiques at EPFL and the Physikalisches Institut at the University of Freiburg for kind hospitality and support. The work of M. H-.V. has been supported by the European Union Grant No. FP7 ITN INVISIBLES (Marie Curie Actions, Grant No. PITN-GA-2011-289442), COST action Grant No. MP1210 (The String Theory Universe) and by the Spanish MINECO Centro de Excelencia Severo Ochoa Programme under Grant No. SEV-2012-0249. S. S. thanks the Kavli Institute for Theoretical Physics at Santa Barbara for hospitality during the initial stage of this project (the visit to KITP was supported in part by the National Science Foundation under Grant No. NSF PHY11-25915). The work of S. S. is supported by the Swiss National Science Foundation. C. S. gratefully acknowledges the kind hospitality and support of the CERN Theory Division and the Instituto de Física Teórica UAM-CSIC while part of this work was being done.

\section{APPENDIX A: A TENSOR IDENTITY}

In dimension $d=2$ any tensor antisymmetric in three or more indices is identically equal to zero. Consider the combination $\hat{k}_{m} \delta_{j}^{i} \delta_{l}^{k}$, where $\hat{k}_{m}$ is a unit vector. By antisymmetrizing it over the lower indices and contracting with $\hat{k}^{m}$ we obtain

$$
\begin{aligned}
0 & =\hat{k}^{m} \hat{k}_{[m} \delta_{j}^{i} \delta_{l]}^{k} \\
& =\delta_{j}^{i} \delta_{l}^{k}-\delta_{l}^{i} \delta_{j}^{k}+\hat{k}^{k} \hat{k}_{j} \delta_{l}^{i}-\hat{k}^{i} \hat{k}_{j} \delta_{l}^{k}+\hat{k}^{i} \hat{k}_{l} \delta_{j}^{k}-\hat{k}^{k} \hat{k}_{l} \delta_{j}^{i} .
\end{aligned}
$$

Next, we lower the indices and symmetrize in $i$ and $j$. This yields

$$
\begin{aligned}
& 2 \delta_{i j} \delta_{k l}-\delta_{i k} \delta_{j l}-\delta_{i l} \delta_{j k}-2 \delta_{i j} \hat{k}_{k} \hat{k}_{l}-2 \hat{k}_{i} \hat{k}_{j} \delta_{k l}+\hat{k}_{i} \hat{k}_{k} \delta_{j l} \\
& \quad+\hat{k}_{j} \hat{k}_{k} \delta_{i l}+\hat{k}_{i} \hat{k}_{l} \delta_{j k}+\hat{k}_{j} \hat{k}_{l} \delta_{i k}=0 .
\end{aligned}
$$

\section{APPENDIX B: CONVERGENCE OF LOOP INTEGRALS}

In this appendix we use the conventions and notations of Sec. VA. We prove the following statement:

Consider a diagram with $L$ loops and $D_{\text {div }}<0$. Assume that all propagators in the diagram are regular in the sense (19) and that if the momentum and frequency in any of the propagators are frozen, the integral over remaining momenta and frequencies converges (i.e. subdivergences are absent). Then the whole diagram converges. ${ }^{24}$

Proof-CSuppressing the external momenta, the expression for the diagram takes the form

$$
\begin{aligned}
I_{D}= & \int \prod_{l=1}^{L}\left[\mathrm{~d} \omega^{(l)} \mathrm{d}^{2} k^{(l)}\right] \mathcal{F}_{n}(\{\omega\},\{\mathbf{k}\}) \\
& \times \prod_{m=1}^{M}\left[\left(A_{m} \Omega^{(m)}\right)^{2}+B_{m}\left(K^{(m)}\right)^{4}\right]^{-1},
\end{aligned}
$$

where $\mathcal{F}_{n}$ is a polynomial of scaling degree $n ; \Omega^{(m)}$, $K^{(m)}$ are linear combinations of loop frequencies and momenta; and the coefficients $A_{m}, B_{m}$ are strictly positive, $A_{m}, B_{m}>0$. The parameters in (B1) satisfy

$$
4 L+n-4 M=D_{\mathrm{div}} .
$$

It is convenient to transform (B1) into the Schwinger-type representation using

$$
x^{-1}=\int_{0}^{\infty} \mathrm{d} s \mathrm{e}^{-s x} .
$$

This yields

\footnotetext{
${ }^{24}$ Here we are talking about convergence in the UV. Infrared divergences present a separate issue and must be regulated by an IR cutoff; see below.
} 


$$
I_{D}=\int_{0}^{\infty} \prod_{m=1}^{M} \mathrm{~d} s_{m} G(\{s\})
$$

where

$$
\begin{aligned}
G(\{s\})= & \int \prod_{l=1}^{L}\left[\mathrm{~d} \omega^{(l)} \mathrm{d}^{2} k^{(l)}\right] \mathcal{F}_{n}(\{\omega\},\{\mathbf{k}\}) \\
& \times \prod_{m=1}^{M} \exp \left\{-s_{m}\left[A_{m}\left(\Omega^{(m)}\right)^{2}+B_{m}\left(K^{(m)}\right)^{4}\right]\right\} .
\end{aligned}
$$

Let us introduce the parametrization

$$
s_{m}=\bar{s} x_{m}, \quad \sum_{m=1}^{M} x_{m}=1 .
$$

Using the scaling properties of the integrand in (B4) we obtain

$$
G(\{s\})=(\bar{s})^{-L-n / 4} G(\{x\}) .
$$

Substituting into (B3) and introducing UV and IR regulators $\bar{s}_{0}, \bar{s}_{1}$ we obtain

$$
I_{D}^{\mathrm{reg}}=\int_{\bar{s}_{0}}^{\bar{s}_{1}} \mathrm{~d} \bar{s}(\bar{s})^{-D_{\mathrm{div}} / 4-1} \tilde{I},
$$

where

$$
\tilde{I}=\int \prod_{m=1}^{M} \mathrm{~d} x_{m} \delta\left(\sum_{m=1}^{M} x_{m}-1\right) G(\{x\})
$$

is the integral over "angles" and we have used the relation (B2) to write the overall power of $\bar{s}$. If $\tilde{I}$ converges, the UV regulator $\bar{s}_{0}$ can be removed, i.e. the diagram is UV finite (recall that $D_{\text {div }}$ is negative ${ }^{25}$ ).

\footnotetext{
${ }^{25}$ Clearly, in this case the diagram exhibits an IR divergence at the upper end of integration over $\bar{s}$.
}

Thus we have to analyze the convergence of $\tilde{I}$. By inspection of (B4) we see that $G(\{x\})$ can have singularities only at the points where some $x_{m}$ vanishes-otherwise the integral over frequencies and momenta in (B4) is damped by the exponentials. ${ }^{26}$ The most dangerous singularity occurs when all $x \mathrm{~s}$, except one, tend to zero,

$$
x_{1} \approx 1, \quad x_{m} \rightarrow 0, \quad m=2, \ldots, M .
$$

The integral over angles in the $\epsilon$-vicinity of this point takes the form

$$
\begin{aligned}
\tilde{I}_{\epsilon} \approx & \int_{0}^{\epsilon} \prod_{m=2}^{M} \mathrm{~d} x_{m} G(\{x\}) \\
= & \int \mathrm{d} \omega^{(1)} \mathrm{d}^{2} k^{(1)} \exp \left\{-\left[A_{1}\left(\omega^{(1)}\right)^{2}+B_{1}\left(k^{(1)}\right)^{4}\right]\right\} \\
& \times \int \prod_{l=2}^{L}\left[\mathrm{~d} \omega^{(l)} \mathrm{d}^{2} k^{(l)}\right] \mathcal{F}_{n}(\{\omega\},\{\mathbf{k}\}) \\
& \times \prod_{m=2}^{M} \frac{1-\exp \left\{-\epsilon\left[A_{m}\left(\Omega^{(m)}\right)^{2}+B_{m}\left(K^{(m)}\right)^{4}\right]\right\}}{A_{m}\left(\Omega^{(m)}\right)^{2}+B_{m}\left(K^{(m)}\right)^{4}},
\end{aligned}
$$

where, without loss of generality, we have identified the frequency and momentum flowing through the first propagator with $\omega^{(1)}, k^{(1)}$. The integral in the second line of (B5) converges. Indeed, it is free from IR divergences, because the integrand is regular at $\omega^{(l)}, k^{(l)} \rightarrow 0$, whereas a UV divergence is absent by assumption. Furthermore, the integrand can be bounded by rational functions, so the total integral grows at most polynomially in $\omega^{(1)}$ and $k^{(1)}$. Then the integral over $\omega^{(1)}, k^{(1)}$ converges as well. ${ }^{27} \mathrm{We}$ conclude that $\tilde{I}_{\epsilon}$, and hence $\tilde{I}$, converges, which completes the proof.

\footnotetext{
${ }^{26}$ Note that this step in the argument relies on the strict positivity of $A_{m}$ and $B_{m}$.

${ }^{27}$ Again, it is important that both $A_{1}$ and $B_{1}$ are strictly positive.
}

[1] J. Polchinski, String Theory (Cambridge University Press, Cambridge, 1998).

[2] K. S. Stelle, Renormalization of higher derivative quantum gravity, Phys. Rev. D 16, 953 (1977).

[3] E. S. Fradkin and A. A. Tseytlin, Renormalizable asymptotically free quantum theory of gravity, Phys. Lett. 104B, 377 (1981); Renormalizable asymptotically free quantum theory of gravity, Nucl. Phys. B201, 469 (1982).

[4] I. G. Avramidi and A. O. Barvinsky, Asymptotic freedom in higher derivative quantum gravity, Phys. Lett. 159B, 269 (1985).
[5] A. Salvio and A. Strumia, Agravity, J. High Energy Phys. 06 (2014) 080.

[6] M. B. Einhorn and D. R. T. Jones, Naturalness and dimensional transmutation in classically scale-invariant gravity, J. High Energy Phys. 03 (2015) 047.

[7] P. Hořava, Membranes at quantum criticality, J. High Energy Phys. 03 (2009) 020.

[8] P. Hořava, Quantum gravity at a Lifshitz point, Phys. Rev. D 79, 084008 (2009).

[9] S. Mukohyama, Hořava-Lifshitz cosmology: a review, Classical Quantum Gravity 27, 223101 (2010). 
[10] T. P. Sotiriou, Hořava-Lifshitz gravity: a status report, J. Phys. Conf. Ser. 283, 012034 (2011).

[11] D. Blas, O. Pujolas, and S. Sibiryakov, Consistent Extension of Hořava Gravity, Phys. Rev. Lett. 104, 181302 (2010).

[12] D. Blas, O. Pujolas, and S. Sibiryakov, Models of nonrelativistic quantum gravity: the good, the bad, and the healthy, J. High Energy Phys. 04 (2011) 018.

[13] D. Blas and S. Sibiryakov, Technically natural dark energy from Lorentz breaking, J. Cosmol. Astropart. Phys. 07 (2011) 026; B. Audren, D. Blas, J. Lesgourgues, and S. Sibiryakov, Cosmological constraints on Lorentz violating dark energy, J. Cosmol. Astropart. Phys. 08 (2013) 039.

[14] D. Blas, M. M. Ivanov, and S. Sibiryakov, Testing Lorentz invariance of dark matter, J. Cosmol. Astropart. Phys. 10 (2012) 057; B. Audren, D. Blas, M. M. Ivanov, J. Lesgourgues, and S. Sibiryakov, Cosmological constraints on deviations from Lorentz invariance in gravity and dark matter, J. Cosmol. Astropart. Phys. 03 (2015) 016.

[15] K. Yagi, D. Blas, N. Yunes, and E. Barausse, Strong Binary Pulsar Constraints on Lorentz Violation in Gravity, Phys. Rev. Lett. 112, 161101 (2014); Constraints on Einstein-aether theory and Hořava gravity from binary pulsar observations, Phys. Rev. D 89, 084067 (2014); 90, 069902 (2014); 90, 069901 (2014).

[16] D. Blas and E. Lim, Phenomenology of theories of gravity without Lorentz invariance: the preferred frame case, Int. J. Mod. Phys. D 23, 1443009 (2014).

[17] S. G. Nibbelink and M. Pospelov, Lorentz Violation in Supersymmetric Field Theories, Phys. Rev. Lett. 94, 081601 (2005); P. A. Bolokhov, S. G. Nibbelink, and M. Pospelov, Lorentz violating supersymmetric quantum electrodynamics, Phys. Rev. D 72, 015013 (2005).

[18] O. Pujolas and S. Sibiryakov, Supersymmetric aether, J. High Energy Phys. 01 (2012) 062.

[19] M. Pospelov and Y. Shang, On Lorentz violation in Hořava-Lifshitz-type theories, Phys. Rev. D 85, 105001 (2012).

[20] G. Bednik, O. Pujolàs, and S. Sibiryakov, Emergent Lorentz invariance from strong dynamics: holographic examples, J. High Energy Phys. 11 (2013) 064; I. Kharuk and S. Sibiryakov, Emergent Lorentz invariance with chiral fermions, arXiv:1505.04130.

[21] S. Janiszewski and A. Karch, Nonrelativistic holography from Hořava gravity, J. High Energy Phys. 02 (2013) 123; String Theory Embeddings of Nonrelativistic Field Theories and their Holographic Hořava Gravity Duals, Phys. Rev. Lett. 110, 081601 (2013).

[22] T. Griffin, P. Hořava, and C. M. Melby-Thompson, Lifshitz Gravity for Lifshitz Holography, Phys. Rev. Lett. 110, 081602 (2013).

[23] T. Fujimori, T. Inami, K. Izumi, and T. Kitamura, Tree-level unitarity and renormalizability in Lifshitz scalar theory, arXiv:1510.07237.

[24] D. Anselmi and M. Halat, Renormalization of Lorentz violating theories, Phys. Rev. D 76, 125011 (2007).

[25] D. Anselmi, Weighted power counting and Lorentz violating gauge theories. I. General properties, Ann. Phys. (Amsterdam) 324, 874 (2009).
[26] D. Blas and S. Sibiryakov, Hořava gravity versus thermodynamics: the black hole case, Phys. Rev. D 84, 124043 (2011).

[27] D. Orlando and S. Reffert, On the renormalizability of Hořava-Lifshitz-type gravities, Classical Quantum Gravity 26, 155021 (2009).

[28] P. Hořava, Spectral Dimension of the Universe in Quantum Gravity at a Lifshitz Point, Phys. Rev. Lett. 102, 161301 (2009); C. Anderson, S. J. Carlip, J. H. Cooperman, P. Hořava, R. K. Kommu, and P. R. Zulkowski, Quantizing Hořava-Lifshitz gravity via causal dynamical triangulations, Phys. Rev. D 85, 044027 (2012).

[29] J. Ambjorn, A. Gorlich, S. Jordan, J. Jurkiewicz, and R. Loll, CDT meets Hořava-Lifshitz gravity, Phys. Lett. B 690, 413 (2010).

[30] T. P. Sotiriou, M. Visser, and S. Weinfurtner, Spectral Dimension as a Probe of the Ultraviolet Continuum Regime of Causal Dynamical Triangulations, Phys. Rev. Lett. 107, 131303 (2011).

[31] D. Benedetti and J. Henson, Spacetime condensation in $(2+1)$-dimensional CDT from a Hořava-Lifshitz minisuperspace model, Classical Quantum Gravity 32, 215007 (2015).

[32] D. Benedetti and F. Guarnieri, One-loop renormalization in a toy model of Hořava-Lifshitz gravity, J. High Energy Phys. 03 (2014) 078.

[33] G. D'Odorico, F. Saueressig, and M. Schutten, Asymptotic Freedom in Hořava-Lifshitz Gravity, Phys. Rev. Lett. 113, 171101 (2014); G. D’Odorico, J. W. Goossens, and F. Saueressig, Covariant computation of effective actions in Hořava-Lifshitz gravity, J. High Energy Phys. 10 (2015) 126.

[34] G. Giribet, D. L. Nacir, and F. D. Mazzitelli, Counterterms in semiclassical Hořava-Lifshitz gravity, J. High Energy Phys. 09 (2010) 009.

[35] D. Nesterov and S. N. Solodukhin, Gravitational effective action and entanglement entropy in UV modified theories with and without Lorentz symmetry, Nucl. Phys. B842, 141 (2011).

[36] M. Baggio, J. de Boer, and K. Holsheimer, Anomalous breaking of anisotropic scaling symmetry in the quantum Lifshitz model, J. High Energy Phys. 07 (2012) 099.

[37] T. P. Sotiriou, M. Visser, and S. Weinfurtner, Phenomenologically Viable Lorentz-Violating Quantum Gravity, Phys. Rev. Lett. 102, 251601 (2009).

[38] D. Blas, O. Pujolas, and S. Sibiryakov, On the extra mode and inconsistency of Hořava gravity, J. High Energy Phys. 10 (2009) 029.

[39] C. Becchi, A. Rouet, and R. Stora, Renormalization of the Abelian Higgs-Kibble model, Commun. Math. Phys. 42, 127 (1975); Renormalization of gauge theories, Ann. Phys. (N.Y.) 98, 287 (1976).

[40] I. V. Tyutin, Gauge Invariance in Field Theory and Statistical Physics in Operator Formalism, Lebedev Institute Report No. N39, 1975.

[41] S. Weinberg, The Quantum Theory of Fields, Vol. II, Modern Applications (Cambridge University Press, Cambridge, 1996).

[42] B.S. DeWitt, Dynamical Theory of Groups and Fields (Gordon and Breach, New York, 1965). 
[43] M. J. G. Veltman, Quantum theory of gravitation, AIP Conf. Proc. C 7507281, 265 (1975).

[44] L. F. Abbott, Introduction to the background field method, Acta Phys. Pol. B 13, 33 (1982).

[45] A. O. Barvinsky and G. A. Vilkovisky, The effective action in quantum field theory: Two loop approximation, in Quantum Field Theory and Quantum Statistics, edited by I. A. Batalin et al. (Hilger, Bristol, 1988), Vol. 1, p. 245.

[46] L.F. Abbott, The background field method beyond one loop, Nucl. Phys. B185, 189 (1981).
[47] P. A. Grassi, Stability and renormalization of YangMills theory with background field method: A Regularization independent proof, Nucl. Phys. B462, 524 (1996).

[48] D. Anselmi, Background field method, Batalin-Vilkovisky formalism and parametric completeness of renormalization, Phys. Rev. D 89, 045004 (2014).

[49] T. P. Sotiriou, M. Visser, and S. Weinfurtner, Lowerdimensional Hořava-Lifshitz gravity, Phys. Rev. D 83, 124021 (2011). 